\title{
Optimal Design of Inlet Passage for Waterjet Propulsion System Based on Flow and Geometric Parameters
}

\author{
Weixuan Jiao, Li Cheng ${ }^{(D,}$, Di Zhang, Bowen Zhang, Yeping Su, and Chuan Wang \\ School of Hydraulic, Energy and Power Engineering, Yangzhou University, Jiangsu, China \\ Correspondence should be addressed to Li Cheng; chengli@yzu.edu.cn and Chuan Wang; wangchuan198710@126.com
}

Received 30 May 2019; Accepted 23 September 2019; Published 24 October 2019

Academic Editor: Antonio Riveiro

Copyright (c) 2019 Weixuan Jiao et al. This is an open access article distributed under the Creative Commons Attribution License, which permits unrestricted use, distribution, and reproduction in any medium, provided the original work is properly cited.

\begin{abstract}
As an important overcurrent component in a waterjet propulsion system, the inlet passage is used to connect the propulsion pump and the bottom of the propulsion ship. The anticavitation, vibration, and noise performance of the waterjet propulsion pump are significantly affected by the hydraulic performance of the inlet passage. The hydraulic performance of the inlet passage directly affects the overall performance of the waterjet propulsion system, thus the design and optimization method of the inlet passage is an important part of the hydraulic optimization of the waterjet propulsion system. In this study, the hydraulic characteristics of the inlet passage in the waterjet propulsion system with different flow parameters and geometric parameters were studied by a combination of numerical simulation and experimental verification. The model test was used to verify the hydraulic characteristics of the waterjet propulsion system, and the results show that the numerical results are in good agreement with the test results. The numerical results are reliable. The hydraulic performance of the inlet passage is significantly affected by the inlet velocity ratio. There is a certain correlation between the hydraulic performance of the inlet passage and ship speed, and the hydraulic performance of the inlet passage is limited by ship speed. The geometric parameters of the best optimization case are as follows: the inflow dip angle $\alpha$ is $35^{\circ}$, the length $L$ is $6.38 D_{0}$, and the upper lip angle is $4^{\circ}$. The optimal operating conditions are the conditions of IVR 0.69-0.87.
\end{abstract}

\section{Introduction}

The waterjet propulsion system propels vessels by a reaction force generated by high-speed jetting of the water stream. Sucking water from the bottom of the vessel, the waterjet propulsion system accelerates the water flow by the propulsion pump and obtains thrust by the change of momentum. Waterjet propulsion system has been widely used in civil and military applications because of its high system efficiency, strong anticavitation ability, excellent maneuverability, stable operation, low noise, etc. $[1,2]$. In last decades, the demand for waterjet propulsion has gradually increased. Many experts have done a lot of work on the simulation of the waterjet propulsion system [3-5], the navigation test $[6,7]$, the interaction between the waterjet propeller and the hull $[8,9]$, and the overall design of the waterjet propulsion system $[10,11]$ and obtained a number of useful results.
However, the waterjet propulsion technology still has the following disadvantages: in shallow water navigation, the waterjet propulsion system is prone to the risk of inhaling gravel. For ships that frequently sail in shallow waters, in order to prevent debris from entering the waterjet propulsion pump and damaging the waterjet propulsion system, the intake grid is usually installed at the inlet of the inlet passage to ensure the normal operation of the propulsion system [12].Wang et al. [13] conducted a numerical simulation study on the effect of inlet grid on duct flow performance and waterjet propulsion performance. The study indicates that inlet grids can reduce the duct flow performance and also reduce waterjet propulsion performance. Chang et al. [14] employed the CFD method to make a numerical simulation of the flow through a whole waterjet propulsion system and obtained the precise hydrodynamic force of waterjet intake grid. The real reason why the grid was broken is provided in the paper. 
The steering device is mounted downstream of the nozzle, which can deflect the jet in order to create steering and reversing forces. However, the traditional steering device is insensitive and has poor hydrodynamic performance [15]. Fang et al. [16] and Pandey and Hasegawa [17] used simulation and experiment methods to predict and analyze the maneuvering performances and thrust characteristics of vessels, respectively. Nowadays, there are many researches on the steering device of the waterjet propulsion system $[15,18]$. The fluidic thrust vector control technologies in the aviation field also provides a new research direction for the reversing device of waterjet propulsion $[19,20]$.

Due to the influence of wave parameters, length of the ship, wind resistance on the hull, etc., the stall will occur when the ship is running. There are two types of ship stalls, including wind wave stall and rotating stall [21, 22]. Cheng and Qi [23] found that there is a rotating stall region, the socalled unstable head curve, when many propulsion pumps are operated. This region should be usually avoided for the risk of instabilities during pump starting and operation. Xia et al. [24] analyzed the rotating stall at a low flow rate and suppressed it with separators. The results shows that the flow pattern near the inlet of the propulsion pump and the velocity distribution in the passage are improved when a separator is installed.

The waterjet propulsion pump system is mainly composed of inlet passage, waterjet propulsion pump, nozzle, and so on. The waterjet propulsion pump is the core part of a waterjet propulsion system. Tan et al. [25, 26], He et al. [27], and Wang et al. [28] have done relevant work on the hydraulic performance of mixed-flow pumps and centrifugal pumps. The research results can provide guidance for the optimization of waterjet propulsion pumps. The flow pattern, especially vortex structures like tip leakage vortex, is of crucial importance to the performance of waterjet pump. Liu and Tan [29, 30] and Hao and Tan [31] have done a lot of research on the evolution and development of tip leakage vortex and obtained a number of useful results which can provide theoretical guidance for the further study of tip leakage vortex. The inlet passage is used as an overflow passage connecting the propulsion pump and the bottom of the propulsion ship. The anticavitation, vibration, and noise performance of the waterjet propulsion pump are significantly affected by the hydraulic performance of the inlet passage. Previous studies [10, 32, 33] have shown that the power loss at the intake of the waterjet propulsion system accounts for $7 \% \sim 9 \%$ of the total power of the system. The hydraulic loss of the inlet passage mainly includes the flow separation loss of the inlet, the constriction loss of the flow channel, the blockage loss, and the friction loss. Ding and Wang [34] and Wang et al. [35] performed numerical simulation calculations on flow loss of inlet passage of marine waterjets and energy loss of centrifugal pump, respectively. Jung et al. [36] made stereoscopic particle image velocimetry measurements in a wind tunnel using a prototype waterjet model. They observed a pair of counter-rotating vortices in the mean velocity field at the nozzle exit, due to the variation in intake geometry from a rectangular to a circular section and because of the sudden curvature change on the lip side. Gong et al. [37] used vehicle-mounted three-dimensional underwater particle image velocimetry (PIV) device is in a towing tank to measure the velocity distribution of the inlet passage of a waterjet ship model in a self-propulsion test. The results could help to establish the design requirements for a waterjet-propelled ship type.

The design and optimization method of the inlet passage is an important part of the hydraulic optimization of the waterjet propulsion system, and its hydraulic performance directly affects the overall performance of the waterjet propulsion system [38-40]. The uneven outflow of the inlet passage will cause periodic pulsation of the propulsion pump, induce vibration, and reduce the efficiency of the propulsion pump. Reasonable design and optimization of the inlet passage is conducive to improving the efficiency of the waterjet propulsion system. Therefore, it is of great significance to design and optimize the inlet passage, shorten the design cycle, and improve the hydraulic performance of the waterjet propulsion system. In this study, based on CFD simulation and experiment, the hydraulic optimization of the inlet passage of the waterjet propulsion system was carried out by using CFX software. The hydraulic performance and internal flow law of the inlet passage were analyzed. The hydraulic performance of the inlet passage is optimized based on flow parameters such as inlet velocity ratio and ship speed and geometric parameters such as length, inflow dip, and lip angle configuration.

\section{Numerical Calculation}

2.1. Numerical Model. As shown in Figure 1, the inlet passage of waterjet propulsion system can be divided into six components: the horizontal straight pipe section, the bent section, the inclined straight pipe section, the slope section, the lip, and the impeller shaft. A well-designed inlet passage needs to ensure that the six components are connected smoothly and without sudden change and that the sections from the intake surface to the outlet of the inlet passage shrink continuously.

In order to design the inlet passage with lower resistance coefficient and reduce the hydraulic loss of the inlet passage, the following principles should be followed in the design of inlet passage: (1) the hydrodynamic performance of the inlet passage should be guaranteed to be superior; (2) the inlet passage should be adapted to the hull structure size and meet the installation requirements of the waterjet propulsion system; (3) high efficiency and short cycle in the design of the inlet passage should be guaranteed. As shown in Figure 2, the two-dimensional geometric model of the inlet passage is associated with thirteen parameters, where $L_{1}$ denotes the control parameter of the horizontal straight line section, $L_{2}$ and $L_{3}$ denote the control parameter of the curve section, $L_{5}$ and $L_{6}$ denote the control parameter of the slope curve section, $L_{7}$ denotes the control parameter of the length of the horizontal inlet, $L_{8}$ and $L_{9}$ denote the control parameter of the lip curve section, $L_{4}$ and $L_{10}$ denote the 


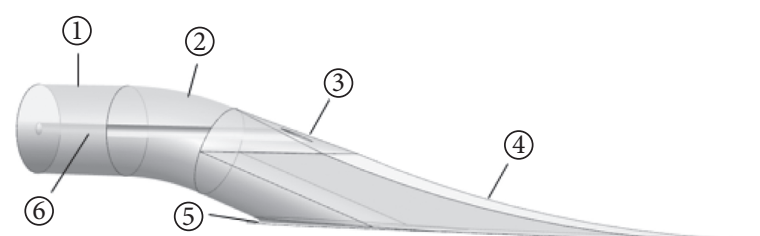

Figure 1: 3D model of inlet flow channel. * (1), horizontal straight pipe section; (2), bent section; (3), inclined straight pipe section; (4), slope section; (5), lip; (6), impeller shaft.

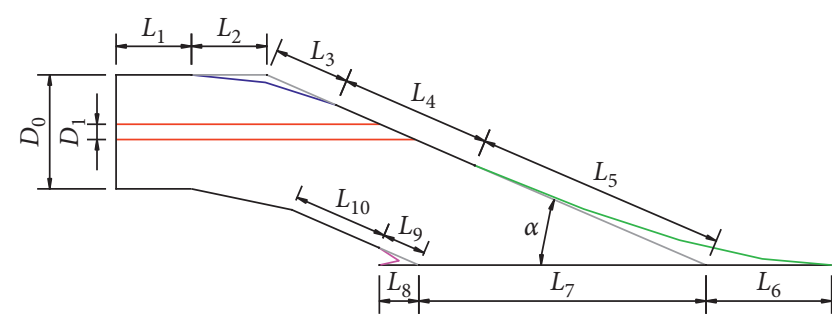

FIGURE 2: 2D structure sketch of inlet passage.

control parameter of the inclined straight line section. $D_{0}$ denotes outlet diameter of the inlet passage, and $D_{1}$ denotes diameter of the impeller shaft. Among them, $L_{5}$ and $L_{9}$ control slope and lip to be, respectively, tangent to the inclined straight line section. $L_{6}$ and $L_{8}$ control slope and lip to be, respectively, tangent to the inlet section. Table 1 presents the basic geometrical parameters of the inlet passage.

2.2. Control Equations and Turbulence Model. Reynolds-averaged $\mathrm{N}$-S equation and continuity equation were selected to describe the 3D incompressible viscous flow. The continuity equation is as follows:

$$
\frac{\partial u_{i}}{\partial x_{i}}=0
$$

The momentum conservation equation is as follows:

$$
\frac{\partial\left(\rho u_{i}\right)}{\partial t}+\frac{\partial\left(\rho u_{i} u_{j}\right)}{\partial x_{j}}=F_{i}-\frac{\partial p}{\partial x_{i}}+\frac{\partial}{\partial x_{j}}\left[\mu\left(\frac{\partial u_{i}}{\partial x_{j}}+\frac{\partial u_{j}}{\partial x_{i}}\right)\right] \text {, }
$$

where $\rho$ represents the water density $\left(\mathrm{m}^{3} / \mathrm{s}\right), u_{i}$ and $u_{j}$ represent the velocity component of fluid in the $i$ and $j$ directions $(\mathrm{m} / \mathrm{s}), t$ represents the time $(\mathrm{s}), p$ represents the pressure $(\mathrm{Pa}), F_{i}$ represents the volume force component in the $i$ direction $(\mathrm{N}), \mu$ represents the dynamics viscosity coefficient, and $x_{i}$ and $x_{j}$ represent the coordinate component.

In order to solve the $\mathrm{N}-\mathrm{S}$ equations, the turbulence model was introduced into numerical calculation to make the equation closed. The $k-\varepsilon$ and $k-\omega$ are two commonly used turbulence models in calculation. Although the turbulence model can predict turbulent motion, the simulation results of different turbulence models will be slightly different. In order to select a more suitable turbulence model, the numerical simulations were carried out for the three commonly used turbulence models Standard $k-\varepsilon$, RNG $k-\varepsilon$, and SST $k-\omega$. Comparing the results of calculations employing different turbulence models with the experimental results [1], the results show that the SST $k-\omega$ model is more consistent with the experimental results. Besides, the convergence accuracy of the calculation employing SST $k-\omega$ model is higher. The SST $k-\omega$ turbulence model is a viscous two-equation turbulence model established on Boussinesq hypothesis, which combined both the advantage of $k-\omega$ model simulation in boundary layer and the advantage of $k-\omega$ model simulation in the free flow region. Hence, the SST (shear stress transport) $k-\omega$ model was used in this study.

2.3. Grid-Sensitivity Analysis. Figure 3 depicts the entire computational domain. Besides the original inlet passage, the additional part is the water body below the hull. Liu et al. [4] simulated the flow control volume of waterjet propulsion by CFD from three dimensions of depth, width, and length, respectively, and compared and analyzed the distribution of physical properties such as flow, power, and thrust and internal flow field velocity. Finally, the results prove that the reasonable size of water body is $30 D_{0} * 10 D_{0} * 8 D_{0}$, where $D_{0}$ is the diameter of inlet passage outlet.

The entire computational domain is generated with hexahedral structured grids by ICEM software. The nearwall mesh of the inlet passage and water body are refined, and the $Y+$ value is controlled within the range of 30-100. The angle of all grids is greater than $18^{\circ}$, and the grid quality is better. The grid and $Y+$ contour of the inlet passage domain are shown in Figure 4.

A grid-sensitivity study was carried out to assess the required grid density. Several grids sizes were considered, ranging from a total number of cells of $2.31 \times 10^{6}$ up to $2.85 \times 10^{6}$. While changing the grid density, the $y+$ values were guaranteed to meet the computational requirements. Table 2 presents grid combination cases for different computational domains.

Figure 5 shows grid sensitivity analysis of the entire computational domain. The hydraulic loss and the pressure coefficient were normalized with respect to its value calculated with Grid 1(grid number $=2.3$ million), where $\Delta h$ represents the hydraulic loss of the inlet passage. The pressure coefficient $C_{p}$ is used to describe the static pressure distribution on the wall of the inlet passage. $C_{p \min }$ represents the minimum cavitation number of the middle section in the inlet passage. From the figure, when the number of grids of the overall calculation domain reaches 2.62 million, the fluctuation of hydraulic loss error and $C_{p}$ error are very small. Theoretically, with the increase of the number and density of grids, the calculation accuracy will generally be improved. However, as the number of grids increases, the requirement for computer configuration increases and the computing speed also slows down. Therefore, the final grid number of entire computational domain is 2619654 .

The formula for the hydraulic loss $\Delta h$ is as follows: 
TABLE 1: Basic geometrical parameters of the inlet passage.

\begin{tabular}{|c|c|c|c|}
\hline Geometric parameter & Value & Geometric parameter & Value \\
\hline Outlet diameter of the inlet passage $D_{0}$ & $220 \mathrm{~mm}$ & Diameter of the impeller shaft $D_{1}$ & $30 \mathrm{~mm}$ \\
\hline$L_{1}$ & $0.68 D_{0}$ & $L_{2}$ & $0.68 D_{0}$ \\
\hline$L_{3}$ & $0.68 D_{0}$ & $L_{4}$ & $1.36 D_{0}$ \\
\hline$L_{5}$ & $2.27 D_{0}$ & $L_{6}$ & $1.14 D_{0}$ \\
\hline$L_{7}$ & $2.59 D_{0}$ & $L_{8}$ & $0.36 D_{0}$ \\
\hline$L_{9}$ & $0.40 D_{0}$ & $L_{10}$ & $0.85 D_{0}$ \\
\hline Dip angle of the inlet passage $\alpha$ & $23^{\circ}$ & & \\
\hline
\end{tabular}

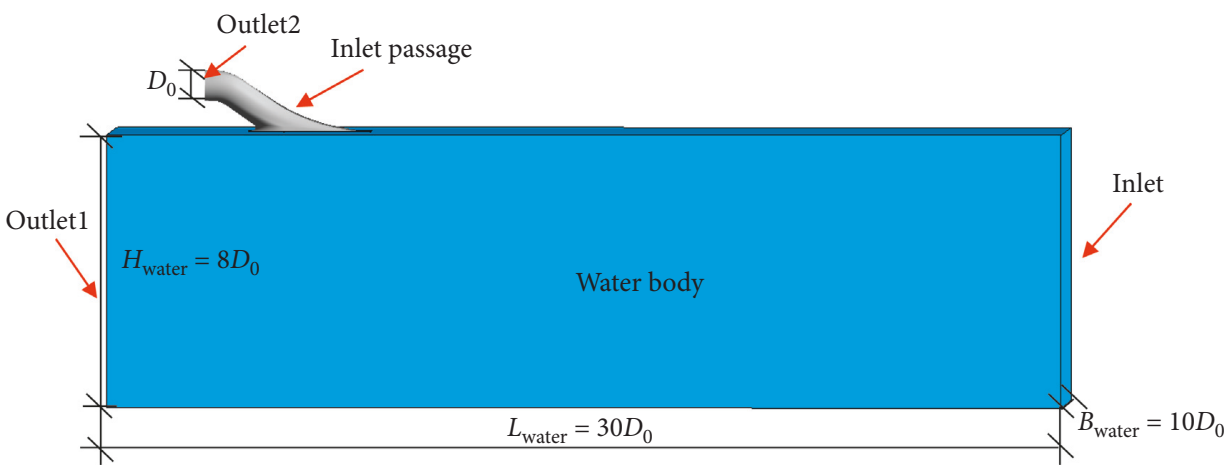

FIgURE 3: Computational domain.

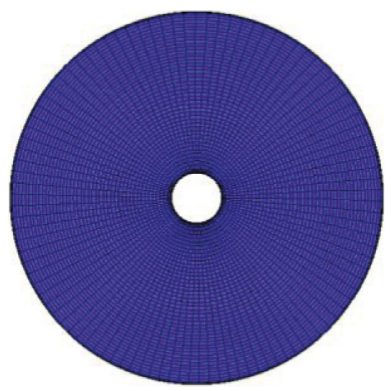

(a)

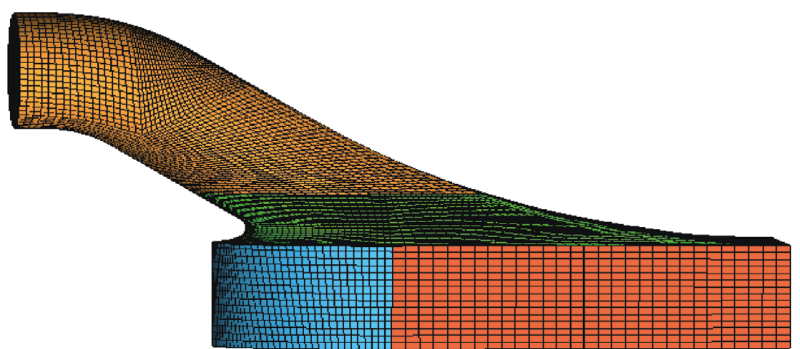

(b)

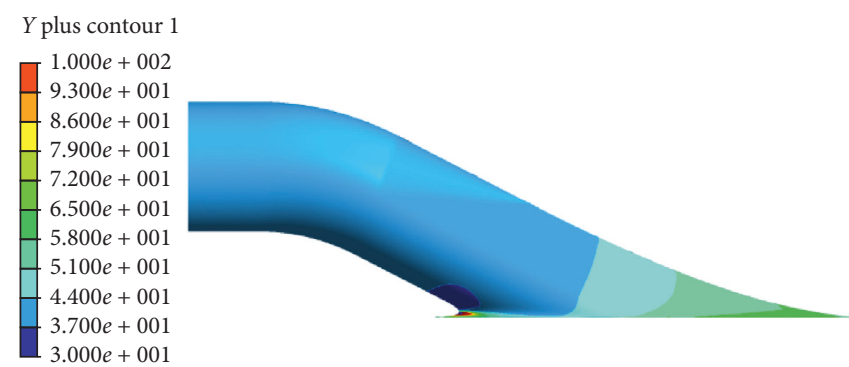

(c)

FIgURE 4: Grid and Y+ contour of the inlet passage domain. (a) Surface mesh of inlet passage outlet. (b) Inlet passage. (c) Y+ contour.

TABLe 2: Grid number.

\begin{tabular}{|c|c|c|c|c|c|}
\hline & 1 & 2 & 3 & 4 & 5 \\
\hline Inlet passage & 1251792 & 1391142 & 1556688 & 1689825 & 1790217 \\
\hline Water body & 1062966 & 1062966 & 1062966 & 1062966 & 1062966 \\
\hline
\end{tabular}




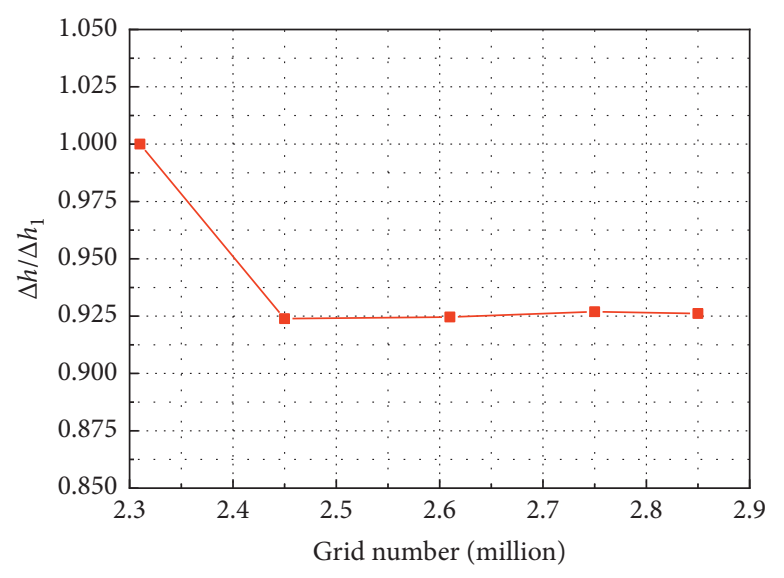

(a)

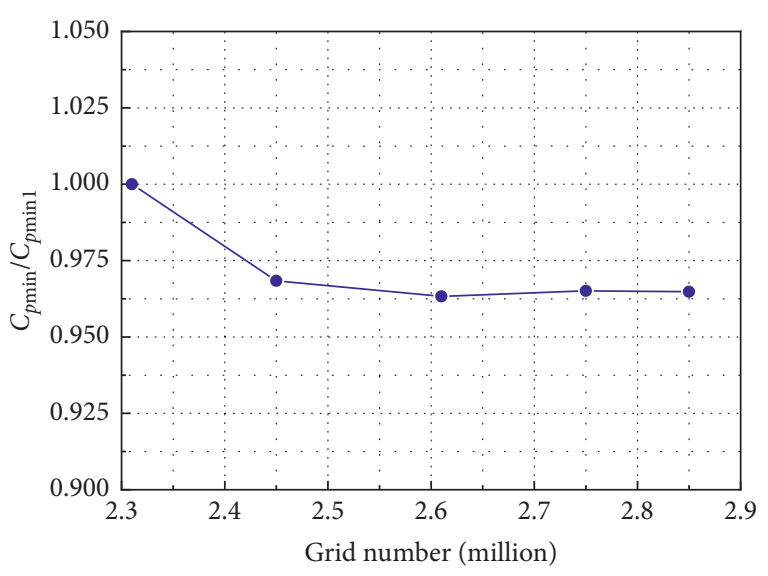

(b)

Figure 5: Grid sensitivity analysis. (a) Hydraulic loss. (b) Pressure coefficient $C_{p}$.

$$
\Delta h=\frac{\Delta P}{\rho g}
$$

where $\Delta P$ is the pressure difference between inlet and outlet of inlet passage (in $\mathrm{Pa}$ ).

The pressure coefficient $C_{p}$ is defined as

$$
C_{p}=\frac{P-P_{\text {ref }}}{(1 / 2) \rho v_{s}^{2}}
$$

where $P$ is instantaneous pressure (in $\mathrm{Pa}$ ), $P_{\text {ref }}$ is reference static pressure (in $\mathrm{Pa}$ ), and $v_{\mathrm{s}}$ is the ship speed (in $\mathrm{m} / \mathrm{s}$ ).

2.4. Boundary Conditions. The inlet of the water body was set as the inlet boundary of the entire computational domain, and the velocity inlet boundary condition was applied at the computational domain inlet. The inlet inflow velocity equals to ship speed $10 \mathrm{~m} / \mathrm{s}$. However, when setting the velocity inlet boundary condition, the Wieghardt equation was used to set the actual inlet velocity due to the influence of hull boundary layer [4]. The nominal turbulence intensities (with value equals to $5 \%$ ) was used at the inlet boundary condition. The outlet of the water body and the inlet passage were set as the outlet boundary. An average static pressure outlet boundary condition was applied with $1 \mathrm{~atm}$ at the water body outlet, and the mass flow rate was adopted as the outlet boundary of the inlet passage. No slip condition was applied at solid boundaries. The convergence precision is set to $10^{-5}$, and the high resolution scheme was used for the convection terms.

The different mass flow rate at the outlet of the inlet passage was set by changing the inlet velocity ratio IVR. The inlet velocity ratio (IVR) is an important parameter to describe the hydraulic characteristics of the inlet passage. IVR refers to the ratio of the ship speed to the averaged axial outflow velocity at the passage outlet. The averaged axial outflow velocity at the passage outlet is an important parameter to describe the flow phenomena in the inlet, where the speed is changed from the ship speed to the pump velocity. IVR is defined as follows:

$$
\operatorname{IVR}=\frac{v_{\text {passage }}}{v_{\mathrm{s}}},
$$

where $v_{\text {passage }}$ is the averaged axial outflow velocity at the passage outlet (in $\mathrm{m} / \mathrm{s}$ ) and $v_{\mathrm{s}}$ is the ship speed (in $\mathrm{m} / \mathrm{s}$ ).

\section{Experimental Setup}

A sketch of test rig is shown in Figure 6, which was designed by Bulten [1]. The model scale test rig was made up of a model scale inlet passage and a cavitation tunnel. The actual waterjet pump is not included in the test rig, but the impeller shaft, with a diameter of $22 \mathrm{~mm}$, is included in the test rig.

The inlet diameter of the model scale inlet passage is $150 \mathrm{~mm}$. The velocity distribution of the impeller plane, at a cross-sectional plane just upstream of the pump, was measured with a 3-hole Pitot-tube. Experiments have been carried out with a constant tunnel speed $V_{\text {tunnel }}$ of $8 \mathrm{~m} / \mathrm{s}$. The tunnel $V_{\text {tunnel }}$ was equal to the ship speed $V_{\text {ship. }}$. Upstream of the inlet an serrated edge is applied to thicken the natural tunnel wall boundary layer. The shape of the edge is selected after an extensive test procedure. During the tests, the growth of the boundary layer thickness and the smoothness of the profiles were evaluated.

Figure 7 shows the comparisons between numerical and experimental results of the axial velocity distribution at the inlet passage outlet. The experiments and numerical simulations were carried out under the condition with the IVR of 0.6 . In the experiment, the inlet mass flow of the tunnel was adjusted to obtain the required IVR values. Based on a survey of the available (confidential) experimental and numerical data, it is concluded that within the design space for commercial applications, all possible inlet geometries show more or less the same type of velocity distribution in Bulten's [1] research. According to the comparison between the experimental results and the numerical results in this paper, the distribution trend of the axial velocity is consistent, which shows that the results of numerical simulation are relatively reliable and the SST $k-\omega$ model selected in this paper is credible. 

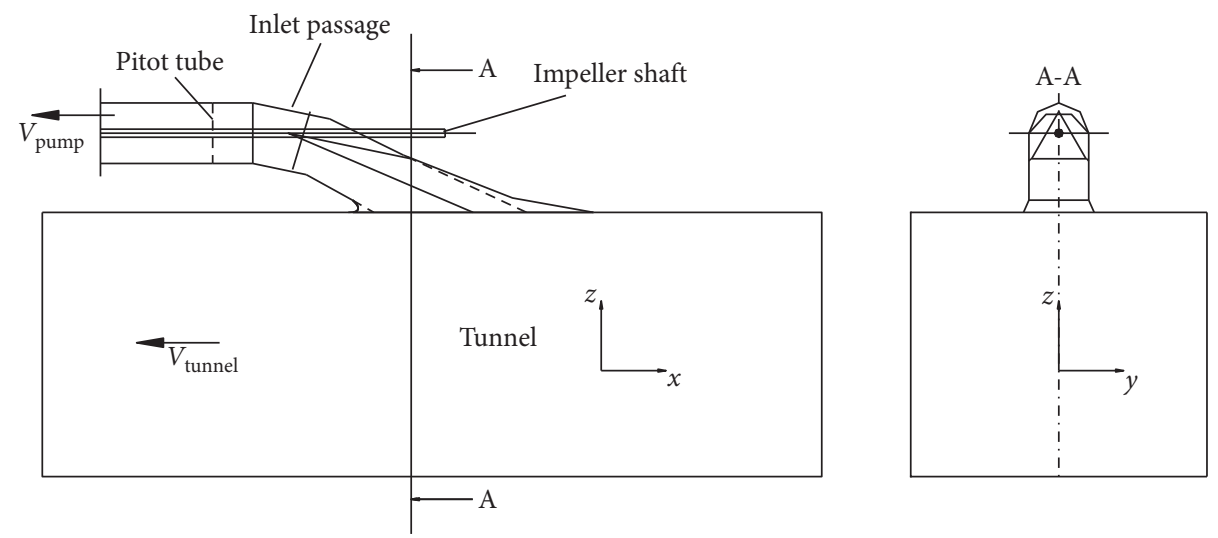

FIGURE 6: Sketch of the test setup with inlet mounted on top of the cavitation tunnel [1].

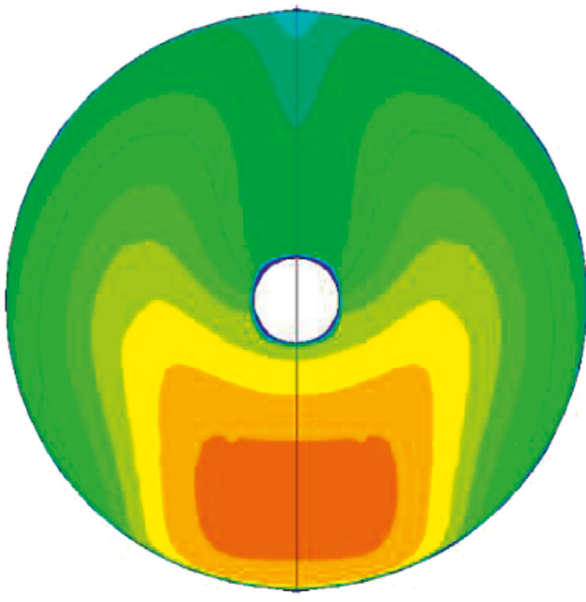

(a)

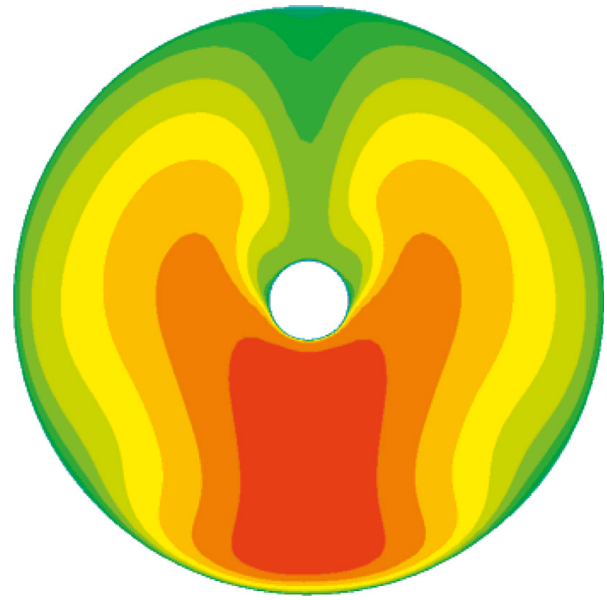

(b)

Figure 7: Comparisons between numerical and experimental results of the axial velocity distribution at inlet passage outlet. (a) Experimental result (Bulten). (b) Numerical result.

\section{Optimal Design of Inlet Passage}

4.1. Optimization Objective Function of Inlet Passage. The flow pattern of the impeller inlet is an important factor affecting the safe and efficient operation of the pump. If the velocity distribution at the impeller inlet section is not uniform, in the process of impeller rotation, the flow will impact the pressure surface or suction surface of the blade, resulting in increased hydraulic loss in the impeller. This is also the reason for the occurrence of flow separation, vortices, and cavitation on the blade surface. Similarly, if the velocity at the impeller inlet section is not perpendicular to that section, it will also change the inlet velocity and the flow angle, resulting in the occurrence of the bad flow patterns near the impeller inlet.

The uniformity of the velocity distribution at the measured section is represented by the axial velocity distribution coefficient $V_{u}$. The axial velocity distribution is the best when the axial velocity distribution coefficient is close to $100 \%$. The weighted-velocity average swirl angle $\theta$ is used to evaluate the outflow conditions of the measured section. The outlet water flow angle of the measured section is better when $\theta$ is closer to $90^{\circ}$. The formula for the axial velocity distribution coefficient $V_{u}$ and weighted-velocity average swirl angle $\theta$ is as follows:

$$
\begin{aligned}
V_{u} & =\left[1-\frac{1}{\overline{u_{a}}} \sqrt{\frac{\sum\left(u_{a i}-\overline{u_{a}}\right)^{2}}{m}}\right] \times 100 \%, \\
\theta & =\frac{\sum_{i=1}^{n}\left[u_{a i}\left(90-\arctan \left(u_{u i} / u_{a i}\right)\right)\right]}{\sum_{i=1}^{n} u_{a i}},
\end{aligned}
$$

where $u_{a i}$ is the axial velocity of each element of the calculated section (in $\mathrm{m} / \mathrm{s}$ ), $\overline{u_{a}}$ is the averaged axial velocity of the calculated section (in $\mathrm{m} / \mathrm{s}$ ), $u_{t i}$ is the tangential velocity of each element of the calculated section (in $\mathrm{m} / \mathrm{s}$ ), and $m$ is the number of cells of the calculated section.

The pressure coefficient $C_{p}$ is used to describe the static pressure distribution on the wall of the inlet passage. At the same time, the pressure coefficient is used to quantitatively analyze the anticavitation ability of the waterjet propulsion system. The inlet passage cavitation performance of the waterjet propulsion system includes two requirements. 
Firstly, there is no cavitation or cavitation in the duct is not obvious. Secondly, the pressure at the inlet passage outlet should not be too low.

Efficiency is an important indicator for evaluating the inlet passage. The efficiency of inlet passage reflects the utilization degree of inflow energy. Figure 8 shows the 24th ITTC definitions of standard locations of the waterjet propulsion system. Section $1 \mathrm{~A}$ in the figure, located $1 D_{0}$ ahead of the inlet tangency, is the imaginary inlet capture area. The recommended inlet capture area of the 24th ITTC $[41,42]$ adopted a semielliptical shape with a long axis length of $1.5 D_{0}$. Yu et al. [43] used the CFD method to simulate the flow field of the waterjet propeller and determined that the shape of the inlet capture area was semielliptical in the range of common working conditions. The flow rate at the inlet capture area is equal to the flow rate at the inlet passage outlet.

In this study, the efficiency of inlet passage is expressed by the total energy ratio of section $1 \mathrm{~A}$ and section 3 . Section $1 \mathrm{~A}$ is a semielliptical inlet capture area, which is located $1 D_{0}$ before the inlet tangency. The long axis length of the semielliptical inlet capture area is $1.5 D_{0}$. Section 3 represents the inlet passage outlet. The formula for the efficiency of inlet passage $\eta_{\mathrm{p}}$ is as follows:

$$
\eta_{\mathrm{p}}=\frac{E_{\text {inlet }}}{E_{\text {capturearea }}} \times 100 \% .
$$

The energy at section $1 \mathrm{~A} E_{\text {capturearea }}$ and section $3 E_{\text {inlet }}$ can be determined by the following equations:

$$
\begin{aligned}
E_{\text {inlet }} & =\frac{1}{2} \rho V_{\text {passage }}^{2}+P_{\text {passage }}, \\
E_{\text {capturearea }} & =\frac{1}{2} \rho V_{1 A}^{2}+P_{1 A},
\end{aligned}
$$

where $P_{\text {passage }}$ denotes the pressure of inlet passage outlet $(\mathrm{Pa}), V_{1 A}$ denotes the velocity of inlet capture area $(\mathrm{m} / \mathrm{s})$, and $P_{1 A}$ denotes the pressure of inlet capture area $(\mathrm{Pa})$.

\subsection{Flow Parameter Optimization}

4.2.1. Inlet Velocity Ratio IVR. The hydraulic performance of the same inlet passage is different under different working conditions. The inlet velocity ratio IVR determines the suction rate and fluid diffusion rate of the inlet passage under a certain working condition. Table 3 shows the calculation parameters of inlet passage under different IVR conditions.

The outflow velocity and pressure of the inlet passage are affected by factors such as the boundary layer of the hull, the shape of the inlet passage, and the disturbance of the impeller shaft, so the distribution of them is uneven. Figure 9 shows the total pressure distribution at the outlet section of inlet passage with different IVRs. The results show that as the IVR increases, the total pressure at the outlet section of the inlet passage increases continuously, the high pressure distribution area expands continuously, and the low pressure distribution area decreases. The total pressure distribution on the left and right sides of the impeller shaft is symmetrical. The upper part of the impeller shaft is a low pressure area, and the lower part of the impeller shaft is a high pressure area. The high pressure area increases with the increase of IVR. With the increase of IVR, the low pressure region gradually shifts to the lower part of the impeller shaft.

Figure 10 shows the velocity distribution at the outlet section of inlet passage with different IVRs. The results show that with the increase of IVR, the velocity at the outlet section of inlet passage increases, the area of high-speed distribution enlarges, and the area of low-speed distribution decreases. The maximum velocity of the outlet section under the condition of IVR equal to 2.38 is 3.67 times of that under the condition of IVR equal to 0.54 . The velocity distribution on the left and right sides of the impeller shaft is symmetrical. The upper part of the impeller shaft is a low velocity area, and the lower part of the impeller shaft is a high velocity area. The high velocity area increases with the increase of IVR. With the increase of IVR, the low velocity region gradually shifts to the lower part of the impeller shaft. With the increase of IVR, the left and right velocity distribution of impeller shaft becomes asymmetric. The main reasons of uneven outflow at the outlet section of the inlet passage are the intake of boundary water flow, the obstruction of the impeller shaft to the water flow, and the influence of the transition section of the inlet passage bend. Boundary water intake depends on the flow drawn from the boundary layer. As the IVR increases, the boundary layer suction ratio increases and the flow rate gradient decreases, which also leads to increased flow uniformity. The impeller shaft has a hindrance to the water flow. There is a narrow low-speed zone around the drive shaft in the figure, which affects the uniformity of the outlet velocity distribution.

Figure 11 shows pressure contour and streamlines in the inlet passage with different IVRs. The results show that, under different IVR conditions, the internal flow pattern of the inlet passage is relatively uniform. However, excessive or too small IVR conditions will result in backflow and flow separation in the flow passage. Due to the special lip structure, the pressure distribution at the lip position changes drastically. It can be seen from the figure that when the height of the flow passage is constant, a long enough slope transition section can ensure that the incoming flow flows into the pump uniformly. If the length of the slope section is too short, the incoming flow will transit unevenly through the passage, which will easily lead to backflow and increase the hydraulic loss of the inlet passage. This indicates that the flow separation should be avoided or reduced when the length of the inlet is fixed.

Figure 12 shows the axial velocity distribution coefficient $V_{u}$ and weighted-velocity average swirl angle $\theta$ at the outlet section of inlet passage with different IVRs. The results show that as the IVR increases, the uniformity of the flow velocity at the outlet section of inlet passage increases. When the IVR is 2.38 , the axial velocity uniformity coefficient at the outlet section of inlet passage is $79.9 \%$, which is $10 \%$ higher than that under the IVR of 0.54 . Although the axial velocity uniformity at the outlet section is poor when the IVR is 0.54 , the weighted-velocity average swirl angle is better. The 


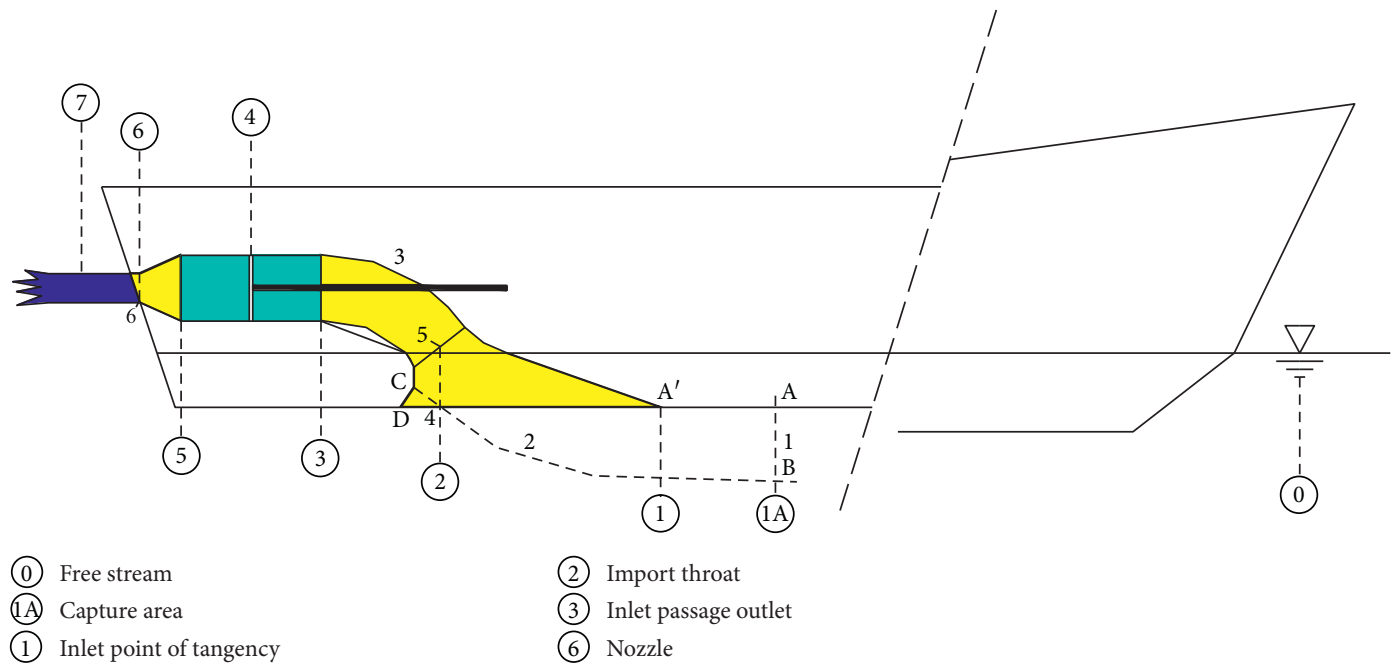

FIgURE 8: 24th ITTC definitions of standard locations of the waterjet propulsion system.

TABLE 3: Calculation parameters of inlet passage.

\begin{tabular}{lcc}
\hline IVR & $V_{\text {tunnel }}(\mathrm{m} / \mathrm{s})$ & $V_{\text {pump }}(\mathrm{m} / \mathrm{s})$ \\
\hline 0.54 & 10 & 5.35 \\
0.59 & 10 & 5.88 \\
0.67 & 10 & 6.67 \\
0.78 & 10 & 7.75 \\
0.94 & 10 & 9.35 \\
1.18 & 10 & 11.76 \\
1.64 & 10 & 16.39 \\
2.38 & 10 & 23.81 \\
\hline
\end{tabular}

results show that with the increase of IVR, the velocity at the outlet section of inlet passage increases, the area of highspeed distribution enlarges, and the area of low-speed distribution decreases. The maximum velocity of the outlet section under the condition of IVR equal to 2.38 is 3.67 times of that under the condition of IVR equal to 0.54 . When the IVR is less than 0.67 , the weighted-velocity average swirl angle of velocity at the outlet section of the inlet passage increases obviously.

Figure 13 shows the sketch of the middle section of the inlet passage. Pressure monitoring points were arranged on the upper and lower walls of the inlet passage. As shown in Figure 14, pressure analysis was carried out on the upper and lower walls of the middle section of the inlet passage. Among them, the abscissa $S / D$ represents the ratio of the distance between the pressure monitoring point in the middle section and the outlet section of the inlet passage $S$ to the outlet diameter $D_{0}$ of the inlet passage. As can be seen from Figure 14(a), when the value of $S / D$ is between 2 and 3 , there is a significant pressure drop on the wall pressure coefficient curve of the inlet passage. This is due to the presence of the impeller shaft, which is more pronounced at high IVR, because the outlet velocity of inlet passage is greatest. Figure 14 (b) presents the pressure coefficient $C_{p}$ curve at the lower wall with different IVRs. It can be seen from the figure that the pressure drops sharply near the lip. Figure 14 shows that as the IVR increases, the pressure on the wall of the inlet passage decreases. There is a greater tendency for pressure drop at the inlet passage outlet, the transition section of the inlet passage bend, and the corners of the lip. Since the pressure at the lower wall is smaller than that at the upper wall under the same IVR, the lower wall is more prone to cavitation than the upper wall. Due to the higher pressure on the middle section of the inlet passage under low IVR conditions, the anticavitation performance of the inlet passage under low IVR operation is better than that of high IVR. Under high IVR conditions, the minimum pressure point at the lip corner of the lower wall moves from the lower edge to the upper edge.

Figure 15 shows the minimum cavitation number $C_{p \min }$ with different IVRs. As the IVR increases, the minimum cavitation number of the middle section rises first and then decreases. As can be seen from the figure, when the value of IVR is between 0.78 and 0.96 , the minimum cavitation number is greater than the critical cavitation number. This indicates that cavitation is less likely to occur in the inlet passage at this time. By comparing the hydraulic performance of the inlet passage under different IVR conditions, it is found that when the IVR is 0.87 , the flow efficiency is $96.2 \%$, and the comprehensive hydraulic performance of the inlet passage is optimal. In summary, the hydraulic performance of the inlet passage is significantly affected by the inlet velocity ratio (IVR). Under low IVR conditions, cavitation is not easy to occur in the inlet passage, and the outflow uniformity is poor. Cavitation is easy to occur in the inlet passage under high IVR condition, and the outflow uniformity of the inlet passage is better. The comprehensive hydraulic performance of the inlet passage is excellent when the IVR is between 0.78 and 0.96 . When the IVR is between 0.78 and 0.96 , the inlet passage of the prototype case is in the optimal operating condition.

4.2.2. Ship Speed. In order to analyze the influence of ship speed on the hydraulic performance of the inlet passage, the uniformity of the outflow velocity distribution, the weighted-velocity average swirl angle of the outflow velocity 


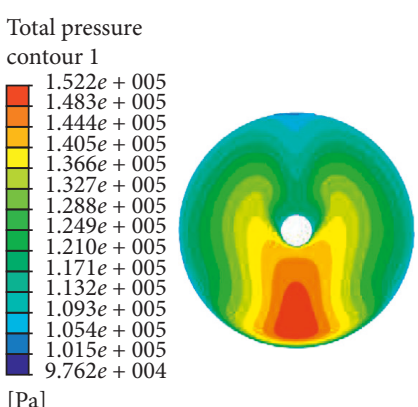

(a)

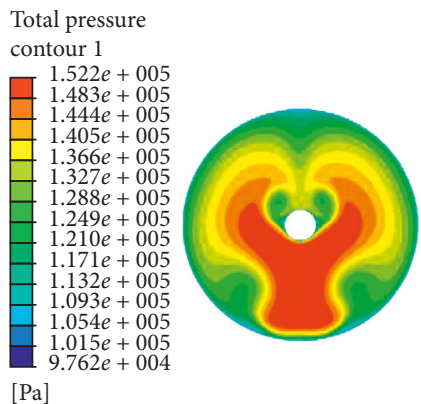

(d)

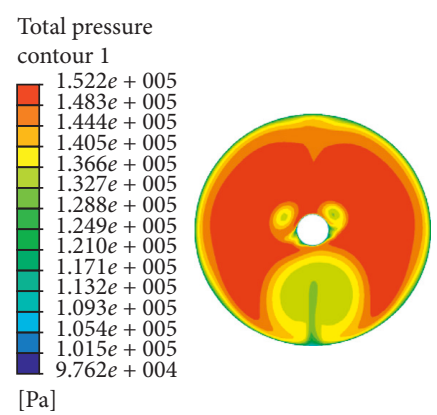

(g)
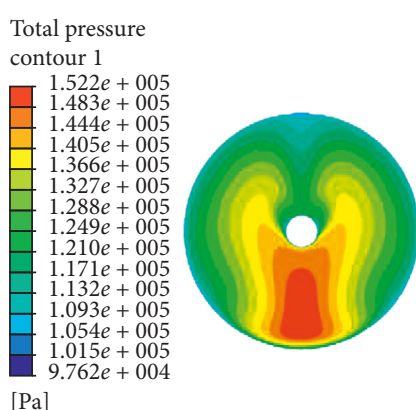

(b)

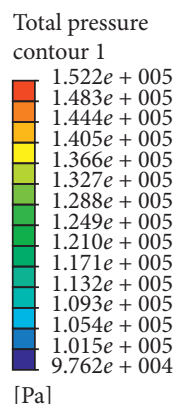

[Pa]

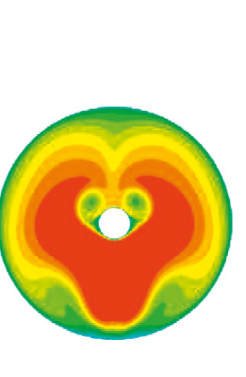

(e)

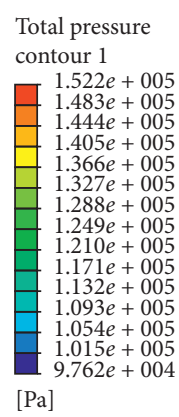

(h)

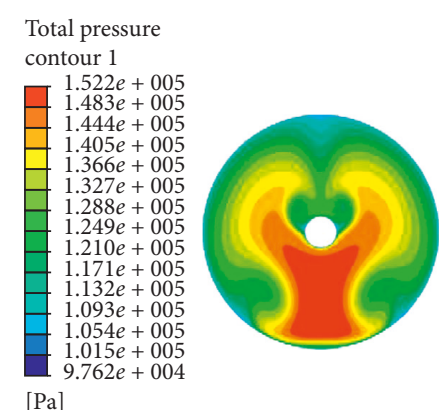

(c)

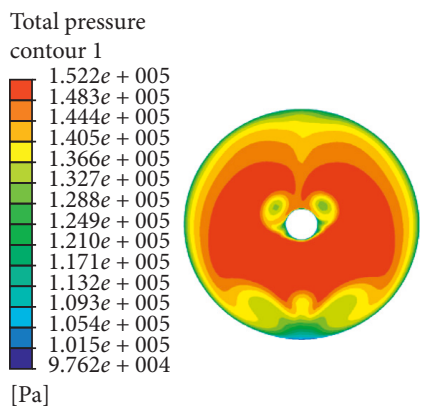

(f)

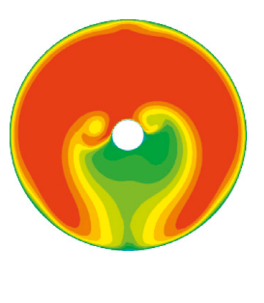

FIGURE 9: Total pressure distribution contours at the outlet section of inlet passage with different IVRs. (a) 0.54 , (b) 0.59 , (c) 0.67 , (d) 0.78 , (e) $0.94,(f) 1.18,(g) 1.64$, and (h) 2.38 .

distribution, and the cavitation performance of the inlet passage at $10 \mathrm{~m} / \mathrm{s}, 12 \mathrm{~m} / \mathrm{s}$, and $15 \mathrm{~m} / \mathrm{s}$ were studied. Figure 16 presents the axial velocity distribution coefficient and weighted-velocity average swirl angle with different ship speeds. As can be seen from Figure 16(a), under the same IVR condition, as the ship speed increases, the velocity distribution uniformity of the inlet passage outlet increases, but the increase is limited, and the maximum growth rate is about $3.7 \%$. This shows that the uniform performance of the inlet passage can be improved when the ship speed is increased, but the effect is limited. Figure 16(b) shows the weighted-velocity average swirl angle of inlet passage outlet increases slightly as the ship speed increases, and the maximum increase is about $1^{\circ}$.

Figure 17 shows the minimum pressure in the middle section of the inlet passage varying with IVR at different speeds. The results show that the longitudinal wall pressure in the inlet passage is greatly affected by the ship speed. As the ship speed increases, the minimum pressure on the wall of the inlet passage decreases as a whole, and the optimal operating point range narrows. Under low IVR and high IVR conditions, the minimum pressure on the wall of the inlet passage is significantly reduced, and the greater the speed, the greater the wall pressure drops. When the ship speed increases to a certain value, the cavitation phenomenon still occurs at the optimal design condition of the inlet passage. The larger the ship speed, the wider the cavitation area, which indicates that there is a ship speed limit when the waterjet propulsion ship is operating under normal conditions.

4.3. Geometric Parameter Optimization. In order to analyze the influence of key geometric parameters on the hydraulic performance and flow characteristics of the inlet passage, three geometric parameters of the length, dip angle, and lip angle of the inlet passage were studied. Research case of geometric parameters is as shown in Table 4. 


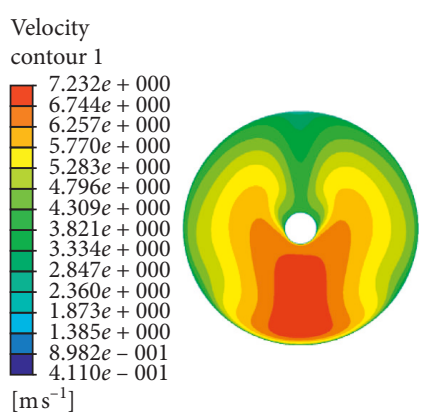

(a)

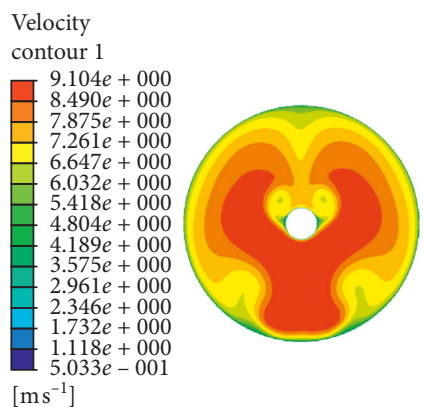

(d)

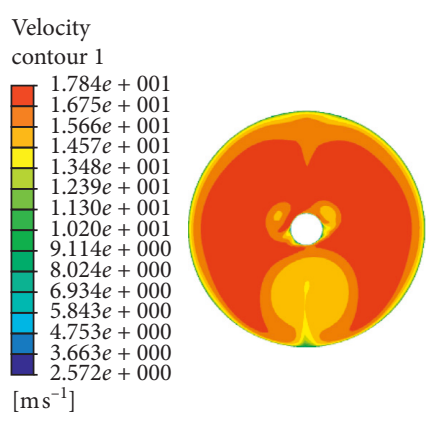

(g)

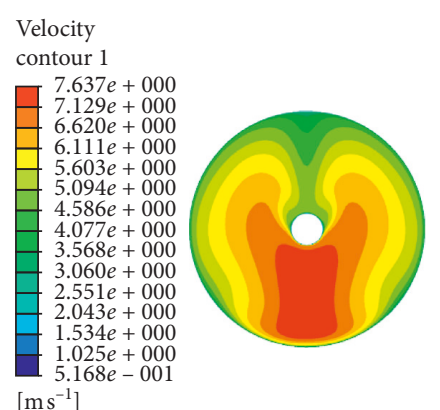

(b)

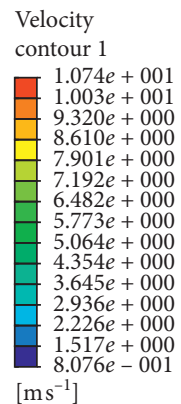

(e)

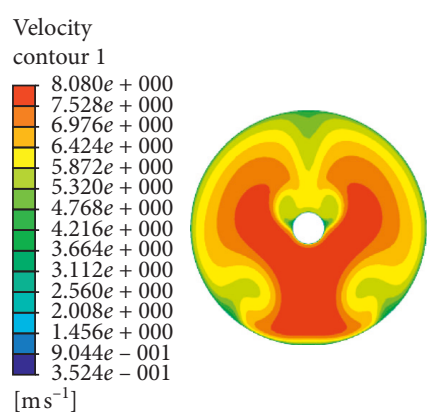

(c)

Velocity

contour 1

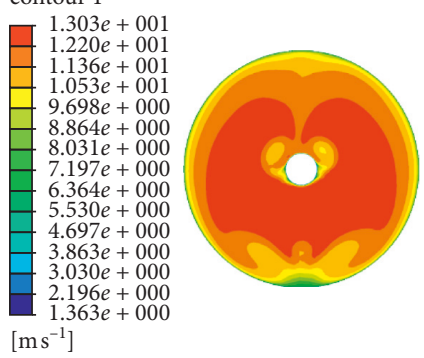

(f)

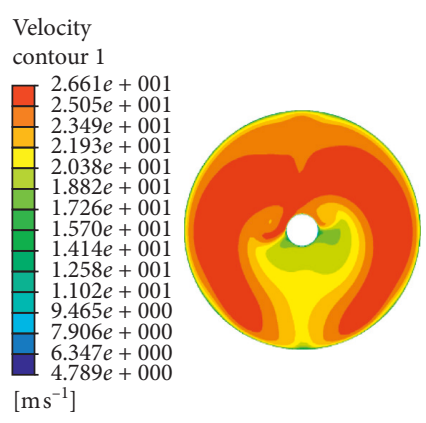

(h)

Figure 10: Velocity distribution contours at the outlet section of inlet passage with different IVRs. (a) 0.5 , (b) 0.59 , (c) 0.67 , (d) 0.78 , (e) 0.94 , (f) $1.18,(g) 1.64$, and (h) 2.38 .

4.3.1. Length. In the case of keeping the height $H$ of inlet passage constant, the length $L$ was changed by changing the spline curve at the slope. Three different length cases were used to study the hydraulic performance of the inlet passage, as shown in Figure 18.

Figure 19 shows the variation of axial velocity distribution coefficient and weighted-velocity average swirl angle at the inlet passage outlet with IVR for different length cases. As shown in Figure 19(a), the uniformity of outflow velocity at the outlet section of the inlet passage in Cases 2 and 3 is much higher than that in Case 1 . The maximum velocity distribution coefficients of Case 2 and Case 3 are both above $82 \%$, which are $2.49 \%$ and $2.34 \%$ higher than those of Case 1 , respectively. When the IVR is less than 0.68 , the velocity uniformity of Case 3 decreases the most. Moreover, when the IVR is equal to 0.54 , the velocity uniformity of Case 3 is the worst among the three cases. The main reason for the poor velocity uniformity of Case 3 under the low IVR condition is that there are some bad flow patterns such as vortices in the inlet passage of Case 3 under the condition of low inlet velocity ratio. Secondly, because the length of the passage is the shortest, the passage cannot adjust the water flow well and eliminate the vortices. Figure 19(b) presents the variation of weighted-velocity average swirl angle at the inlet passage outlet of three cases with IVR. The weightedvelocity average swirl angle at the inlet passage outlet of Case 2 and 3 decreases slightly compared with that of Case 1 . When the IVR is equal to 0.54 , the difference between the weighted-velocity average swirl angle of Case 1 and that of Cases 2 and 3 is the greatest. The weighted-velocity average swirl angles of Case 1 are $2.3 \%$ and $4.0 \%$ higher than those of Cases 2 and 3, respectively. The weighted-velocity average swirl angle of Case 1 and Case 2 decreases with the increase of IVR in general, but the change trend of Case 3 is opposite. Based on the above analysis, it is found that the hydraulic characteristics of the inlet passage outlet of Case 1 and 2 are significantly better than those of Case 3 . The axial velocity distribution coefficient of the Case 2 is generally improved 


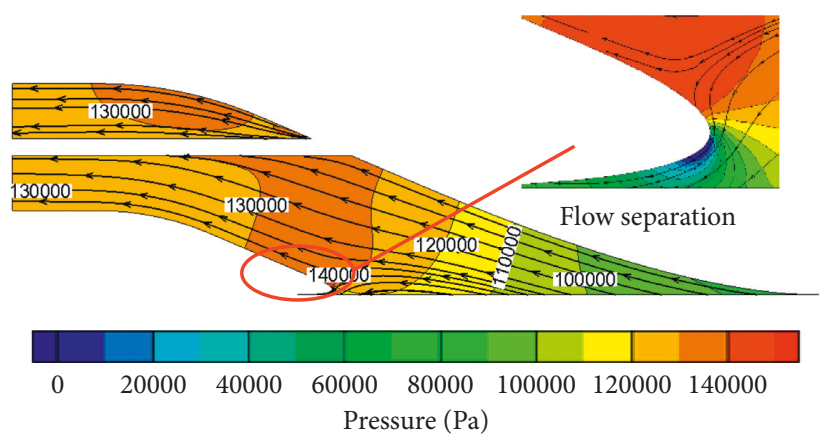

(a)

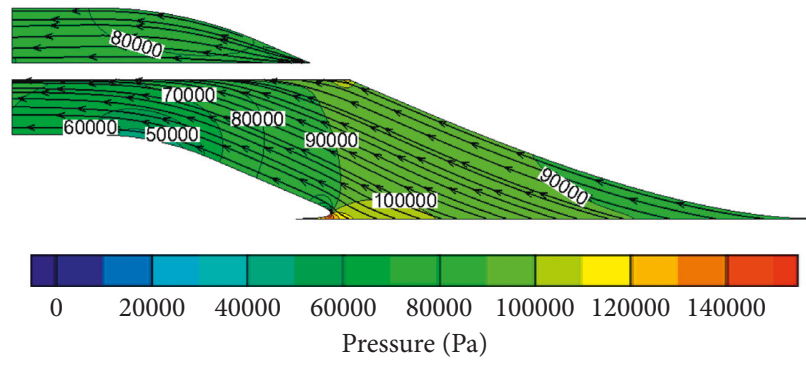

(c)

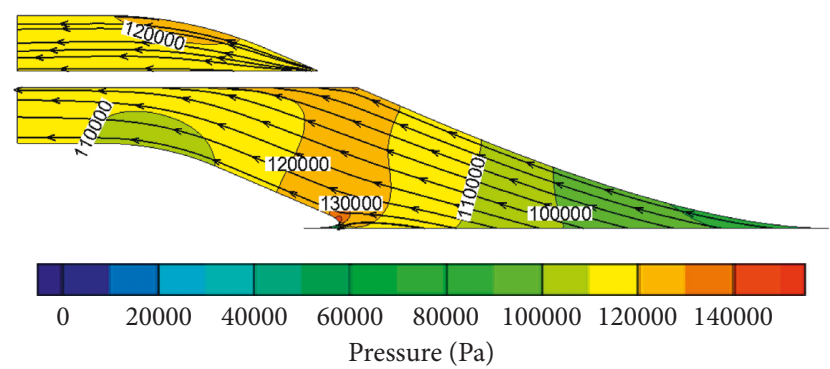

(b)

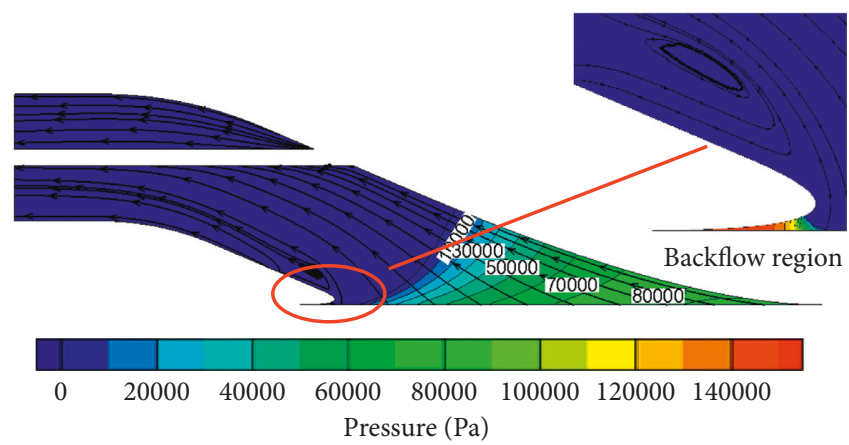

(d)

Figure 11: Pressure contour and streamlines in the inlet passage with different IVRs. (a) 0.54, (b) 0.67, (c) 0.94, and (d) 2.38.

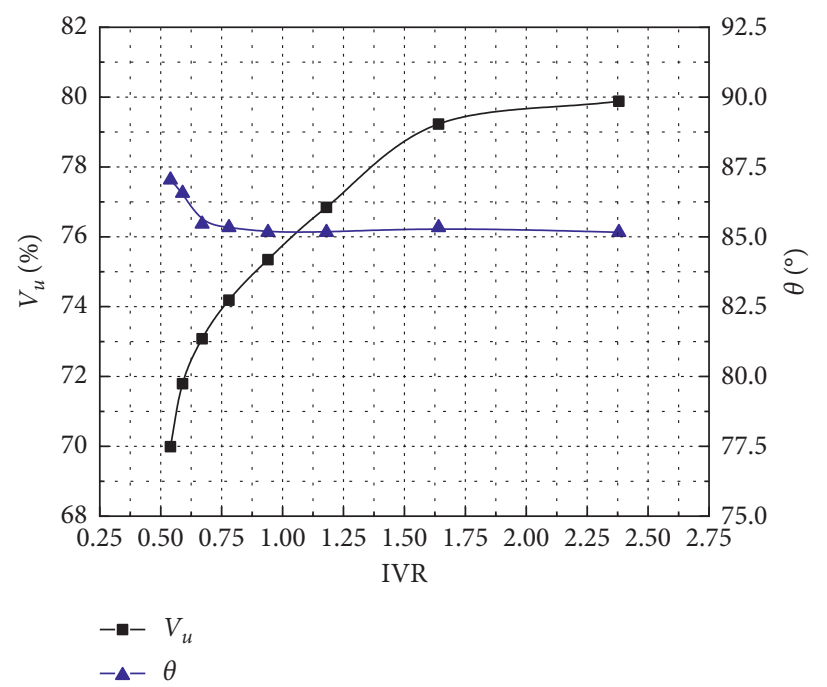

FIGURE 12: Axial velocity distribution coefficient and weighted-velocity average swirl angle with different IVRs.

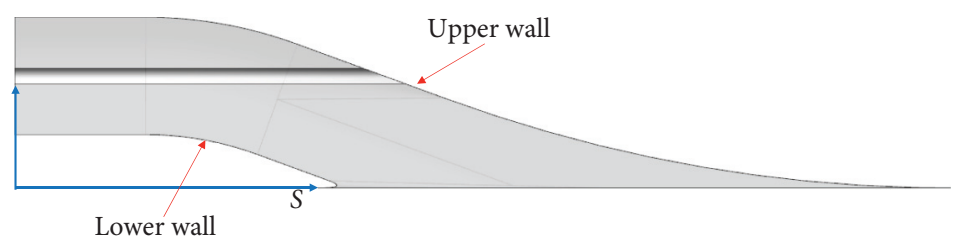

FIGURE 13: Sketch of the middle section of the inlet passage.

relative to the Case 1 , and the maximum difference is $5.11 \%$, and the weighted-velocity average swirl angle decreases slightly.
Figure 20 presents the variation trend of the minimum cavitation number along the wall of the inlet passage with IVR. It can be seen from the figure that the minimum 


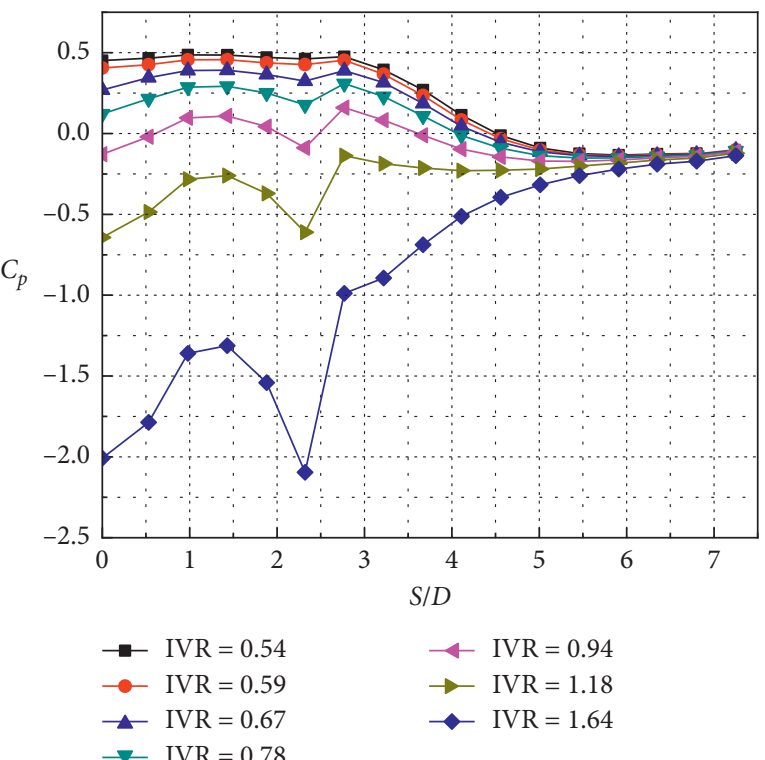

(a)

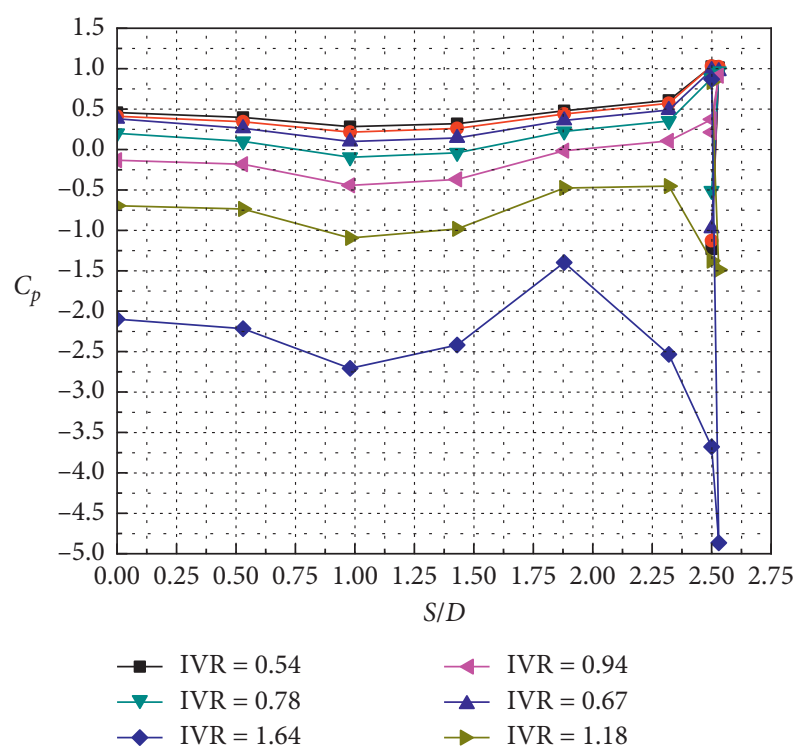

(b)

Figure 14: Pressure coefficient $C_{p}$ curves at the (a) upper and (b) lower walls with different IVRs.

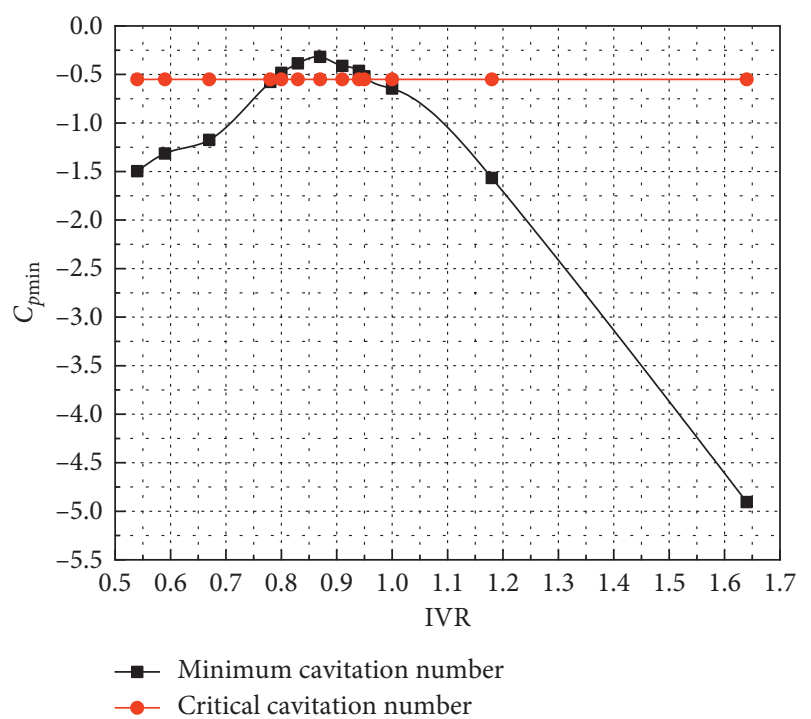

FIGURE 15: Minimum cavitation number $C_{p \min }$ with different IVRs.

cavitation number of the inlet passage wall of Case 3 is basically below the critical cavitation number, which indicates that the anticavitation performance of Case 3 is not good. Under the conditions of low IVR and high IVR, the minimum cavitation number of the inlet passage wall in Case 2 is smaller than that in Case 1 . When the value of IVR is between 0.78 and 0.96 , the anticavitation performance of Case 1 is superior. When the value of IVR is between 0.77 and 0.98 , the anticavitation performance of Case 2 is superior. Therefore, the high efficiency area of Case 2 is wider than that of Case 1.

Figure 21 shows streamlines of the three different inlet passages under the condition of IVR equal to 0.53 . As can be seen from the figure, the streamlines in the inlet passage of Case 1 is uniform, and there is no whirlpool phenomenon. However, in Case 2 and Case 3, there are vortexes and other flow patterns. The whirlpool and reflux phenomena of the Case 3 are serious. Comparing with Figure 19(a), it can be seen that when IVR is equal to $0.53,0.59$, and 0.67 , the flow pattern in the inlet passage is poor, and the adverse flow regimes such as whirlpool and reflux are easy to occur. The above analysis shows that the length $L$ affects the hydraulic performance when other geometric parameters of the duct remain unchanged, that is, the slope curvature should be taken into account in the design of the inlet passage. If the curvature of the slope of the inlet passage is small, the curvature degree of the slope is not obvious, or even close to a straight line, the inlet passage will be prone to undesirable flow phenomena such as whirlpools and the hydraulic loss will be increased.

Table 5 presents the efficiency, axial velocity distribution coefficient, and weighted-velocity average swirl angle of the inlet passage for three cases at the IVR of 0.83 . By comparing the efficiency of the three cases, it can be seen that the length of the inlet passage affects the efficiency of the inlet passage greatly. Reducing the length of the inlet passage will change the slope curvature of the inlet passage. When the curvature of slope exceeds the reasonable range, the hydraulic performance of the inlet passage becomes worse obviously. The efficiency of Case 3 is $24.8 \%$ and $24 \%$ smaller than that of Case 1 and Case 2, respectively, mainly because the flow path length of Case 3 is too small, and whirlpools appear in the inlet passage. In brief, the performance of the inlet passage is significantly affected by its length. The hydraulic performance of the inlet passage for Case 2 is better, and the operating condition IVR is between 0.77 and 0.98 , which broadens the optimal operating conditions of the prototype 


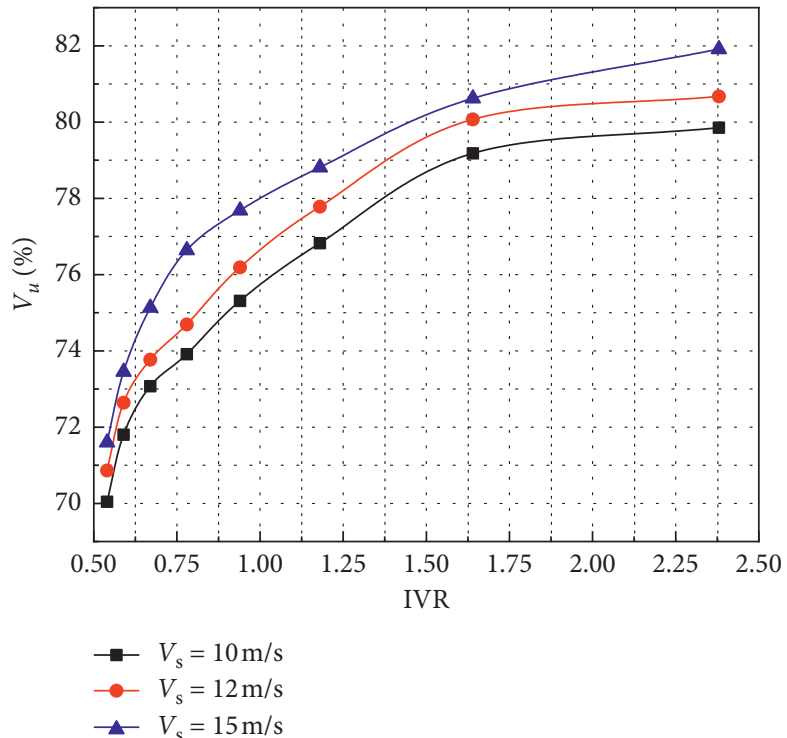

(a)

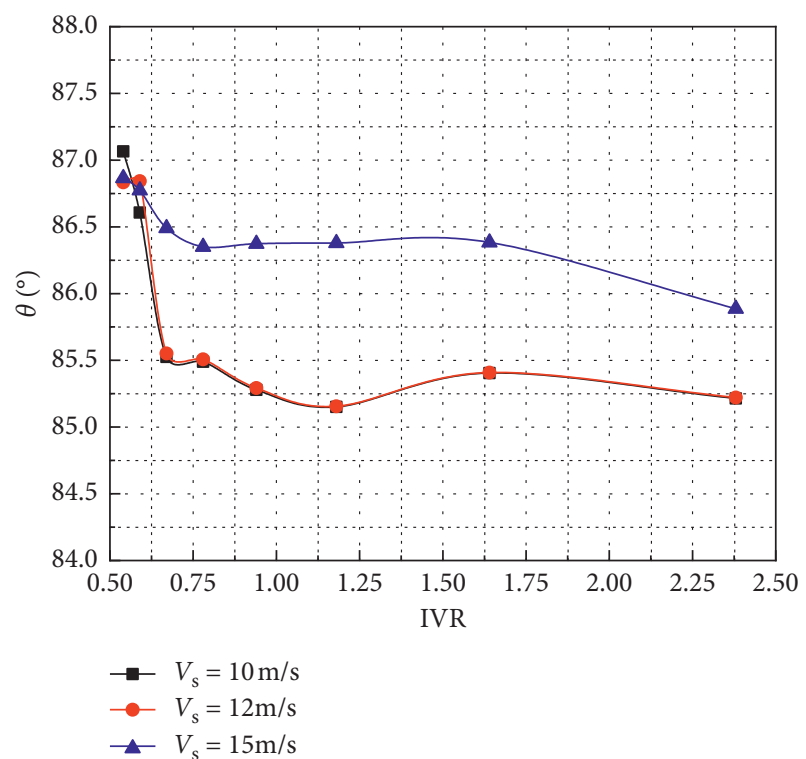

(b)

FIGURE 16: Hydraulic characteristics of inlet passage outlet with different ship speeds. (a) Axial velocity distribution coefficient $V_{u}$. (b) Weighted-velocity average swirl angle $\theta$.

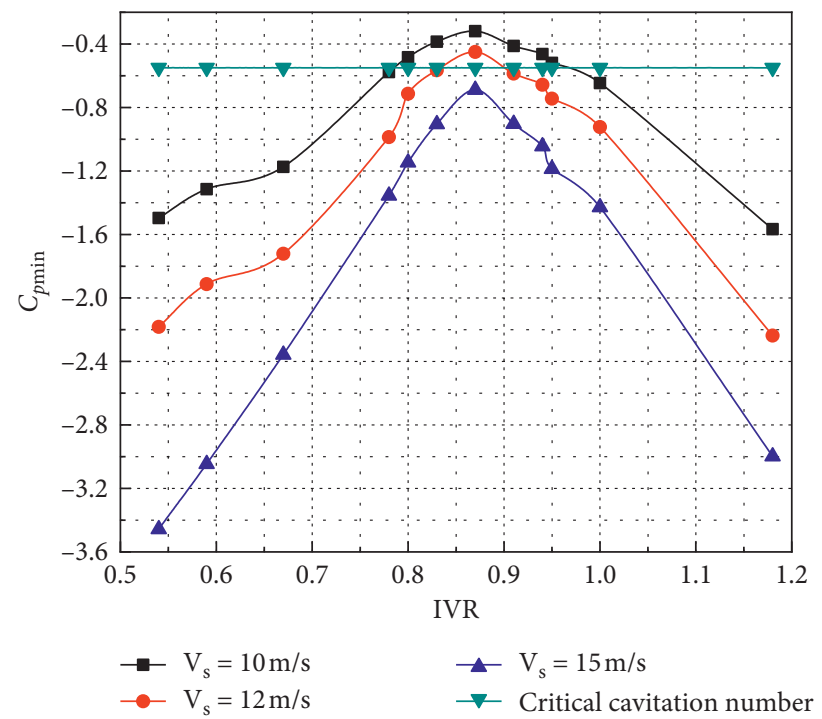

FIgure 17: Minimum cavitation number $C_{p \min }$ with different ship speeds.

case. The slope curvature should be considered in the design to avoid the lack of length and cause undesirable flow phenomena such as whirlpools.

4.3.2. Dip Angle. The length of the inlet passage, the center height of the outlet section, the outlet diameter, and the dip angle of the waterjet propulsion pump system are the key parameters for the design of the inlet passage. The hydraulic performance of the inlet passage for Case 2 is better and the range of optimal operating conditions is wider. Therefore, the optimization is continued on the basis of Case 2, and the hydraulic loss is further reduced by changing the dip angle $\alpha$. Due to the limitation of the size and speed of the waterjet propulsion ship, the adjustment margin of the inlet passage length and the outlet diameter is limited. Therefore, the hydraulic design of the dip angle is particularly important to the inlet passage optimization. Figure 22 shows sketch of the dip angle optimization cases.

Figure 23 shows the variation of axial velocity distribution coefficient and weighted-velocity average swirl angle at the inlet passage outlet with IVR for different dip angle cases. As can be seen from the Figure 23(a), the axial velocity uniformity at the outlet of the inlet passage for Case 6 and Case 7 is significantly improved. As the degree of dip angle increases, the axial velocity uniformity at the outlet of the inlet passage does not increase all the time. The axial velocity uniformity at the outlet of the inlet passage for Case 5 is 
TABLE 4: Research case of geometric parameters.

\begin{tabular}{|c|c|c|c|c|}
\hline \multirow{2}{*}{ Case } & \multicolumn{3}{|c|}{ Geometric parameter } & \multirow{2}{*}{ Note } \\
\hline & Length $(L)$ & Dip angle $(\alpha)$ & Upper lip angle & \\
\hline 1 & $7.45 D_{0}$ & $23^{\circ}$ & $3^{\circ}$ & \\
\hline 2 & $6.38 D_{0}$ & $23^{\circ}$ & $3^{\circ}$ & \\
\hline \multirow[t]{2}{*}{3} & $5.71 D_{0}$ & $23^{\circ}$ & $3^{\circ}$ & $L$ \\
\hline & & & & Length optimization \\
\hline 4 & $6.38 D_{0}$ & $23^{\circ}$ & $3^{\circ}$ & \\
\hline 5 & $6.11 D_{0}$ & $30^{\circ}$ & $3^{\circ}$ & \\
\hline 6 & $5.95 D_{0}$ & $35^{\circ}$ & $3^{\circ}$ & \\
\hline \multirow[t]{2}{*}{7} & $5.76 D_{0}$ & $40^{\circ}$ & $3^{\circ}$ & $L$ \\
\hline & & & & Dip angle optimization \\
\hline 8 & $5.95 D_{0}$ & $35^{\circ}$ & $3^{\circ}$ & \\
\hline 9 & $5.95 D_{0}$ & $35^{\circ}$ & $4^{\circ}$ & \\
\hline 10 & $5.95 D_{0}$ & $35^{\circ}$ & $5^{\circ}$ & \\
\hline 11 & $5.95 D_{0}$ & $35^{\circ}$ & $6^{\circ}$ & \\
\hline \multirow[t]{2}{*}{12} & $5.95 D_{0}$ & $35^{\circ}$ & $7^{\circ}$ & $-L$ \\
\hline & & & & Upper lip angle optimization \\
\hline
\end{tabular}

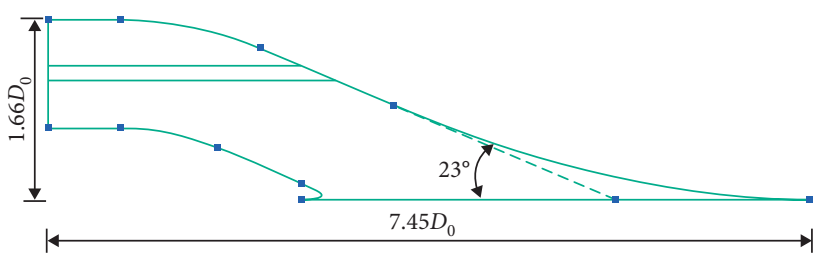

(a)

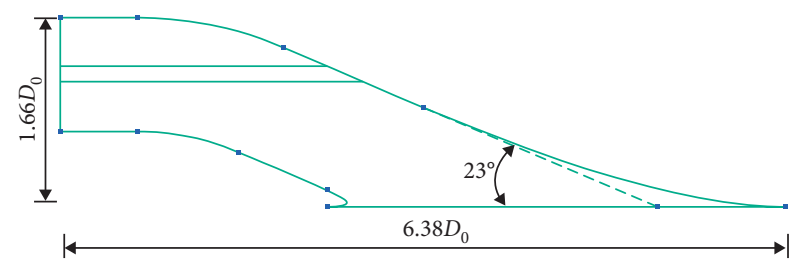

(b)

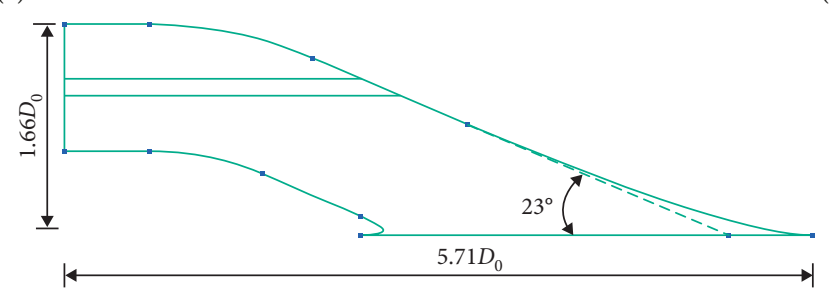

(c)

FIGURE 18: Sketch of the middle section of inlet passages with different lengths. (a) Case 1. (b) Case 2. (c) Case 3.

slightly lower than that for Case 4 . The above analysis shows that there is a better dip angle range which is beneficial to improve the hydraulic performance of the inlet passage. Among the four cases, the outflow uniformity of Case 7 is better than that of Case 6 under different IVR conditions, but the maximum velocity distribution coefficient of Case 6 is higher than that of Case 7 . The highest velocity distribution coefficient of Case 6 is $88.46 \%$. Figure 23(b) shows that the difference for weighted-velocity average swirl angle of the four cases is small, and the weighted-velocity average swirl angle of Case 6 is slightly better than other cases.
Figure 24 presents the variation trend of the minimum cavitation number along the wall of the inlet passage with IVR. It can be seen from the figure that with the increase of the dip angle of the inlet passage, the minimum pressure on the wall of the duct decreases as a whole. The optimum operating condition of the inlet passage shifts to the condition of low IVR, and the operating range decreases gradually with the increase of dip angle. Cavitation phenomena occur on the wall of the inlet passage of Case 7 at different IVRs. The optimal operating condition of Case 6 is the working condition of IVR in the range of $0.73-0.86$. 


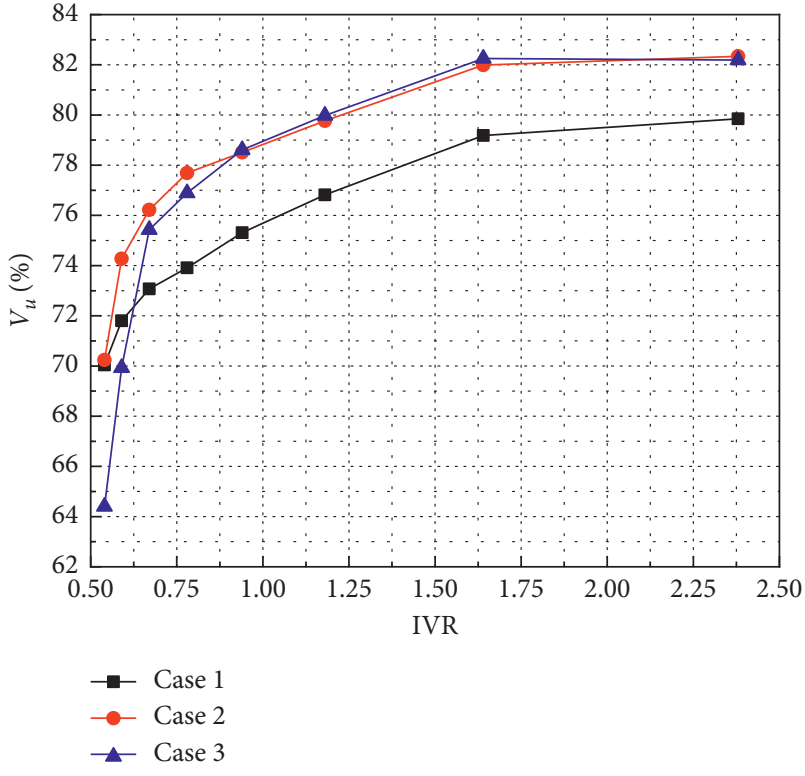

(a)

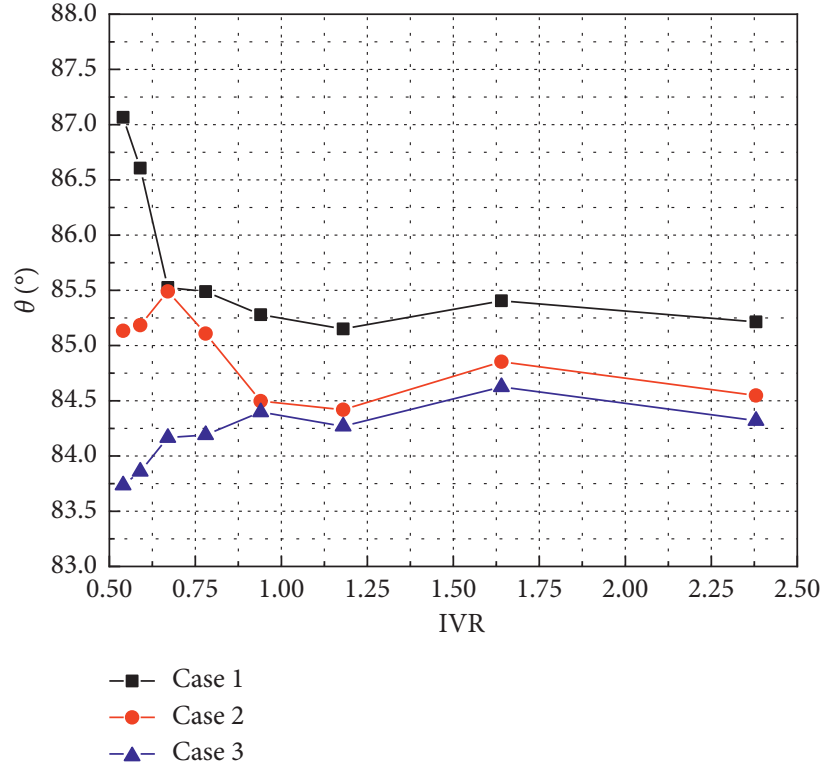

(b)

FIGURE 19: Hydraulic characteristics of inlet passage outlet with different inlet passage lengths. (a) Axial velocity distribution coefficient $V_{u}$. (b) Weighted-velocity average swirl angle $\theta$.

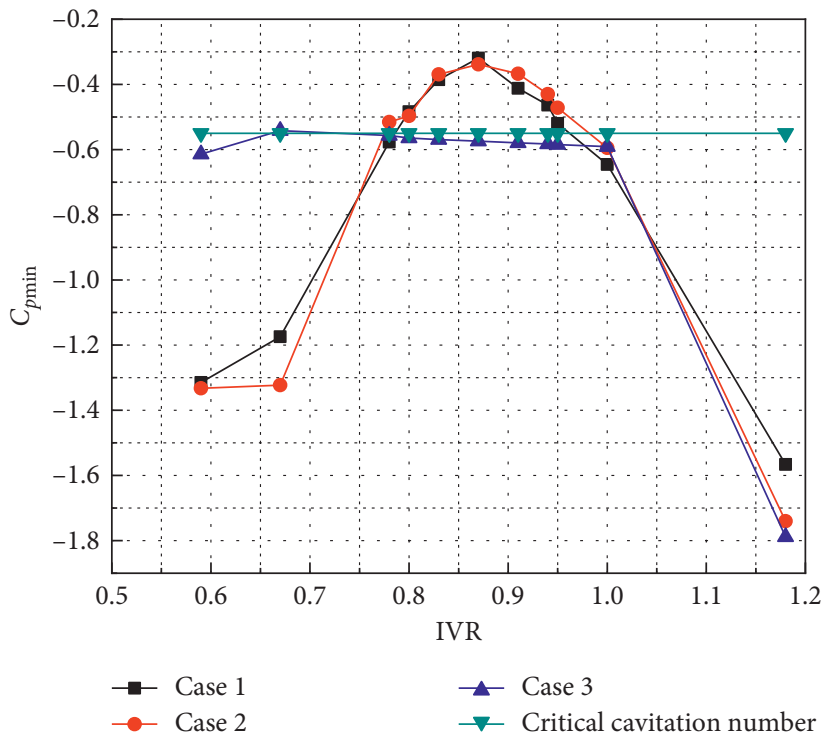

FIgURE 20: Minimum cavitation number $C_{p \min }$ with different inlet passage lengths.

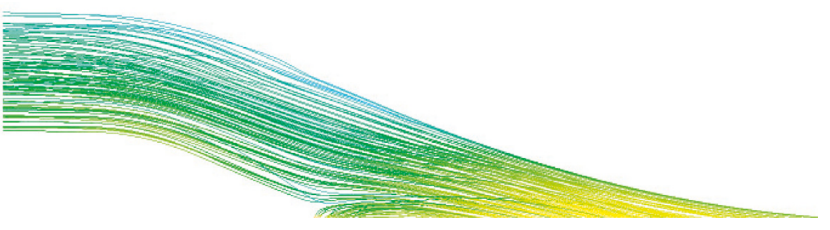

(a)

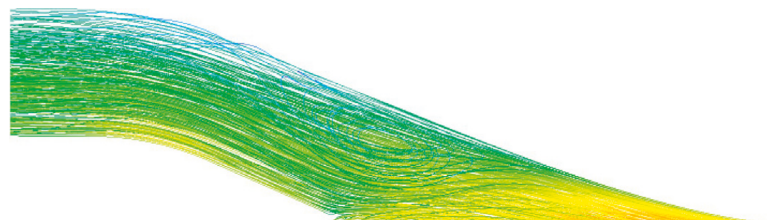

(b)

Figure 21: Continued. 


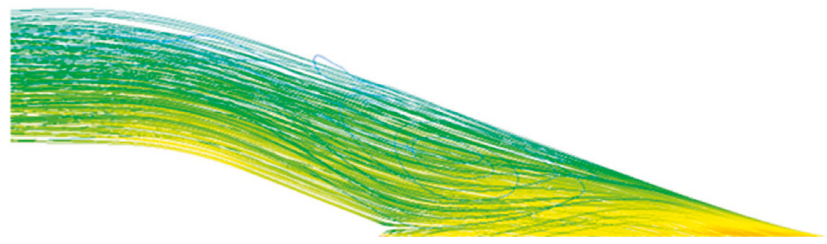

(c)

FIgURE 21: Streamlines of inlet passage under the condition of IVR 0.53. (a) Case 1. (b) Case 2. (c) Case 3.

TABLE 5: Evaluation index of inlet passage.

\begin{tabular}{lccr}
\hline Case & $\eta_{\mathrm{p}}(\%)$ & $V_{u}(\%)$ & $\theta\left(^{\circ}\right)$ \\
\hline Case 1 & 96.2 & 74.6 & 85.4 \\
Case 2 & 95.4 & 78.1 & 84.8 \\
Case 3 & 71.4 & 77.8 & 84.3 \\
\hline
\end{tabular}

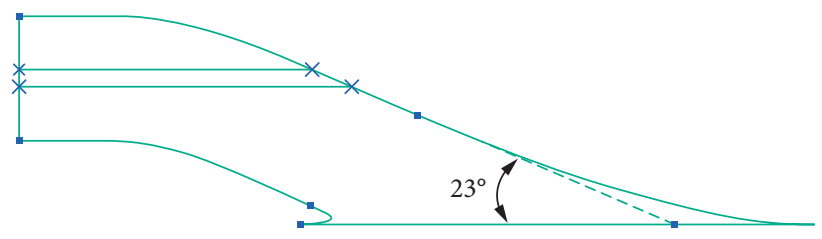

(a)

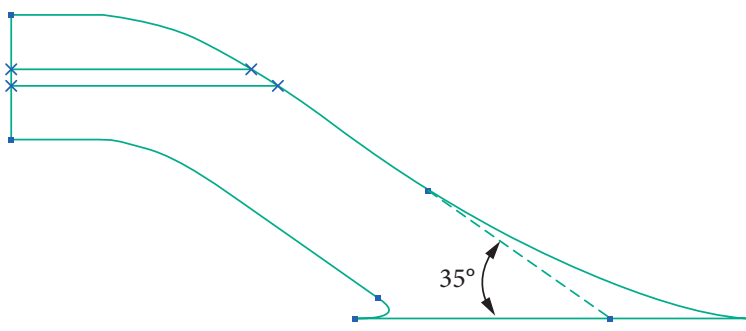

(c)

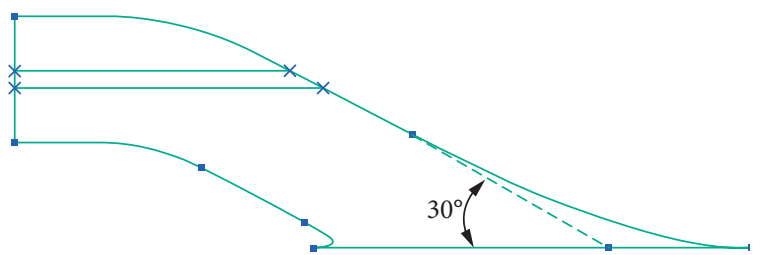

(b)

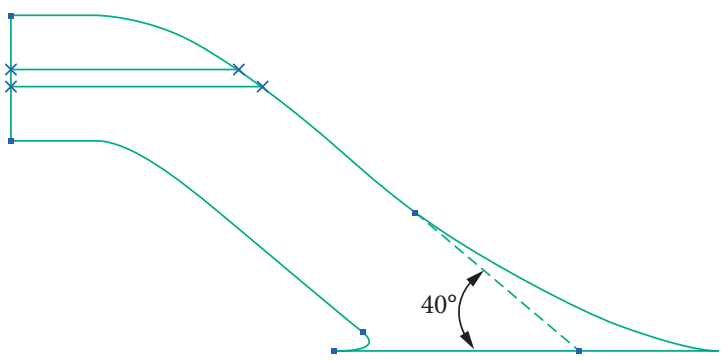

(d)

Figure 22: Sketch of the dip angle optimization cases. (a) Case 4 (b) Case 5. (c) Case 6. (d) Case 7.

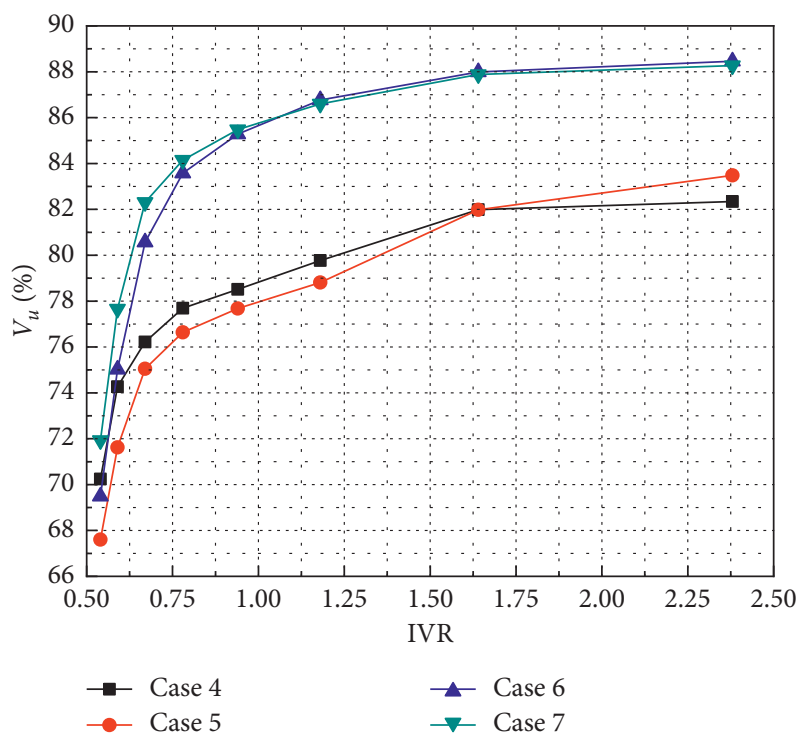

(a)

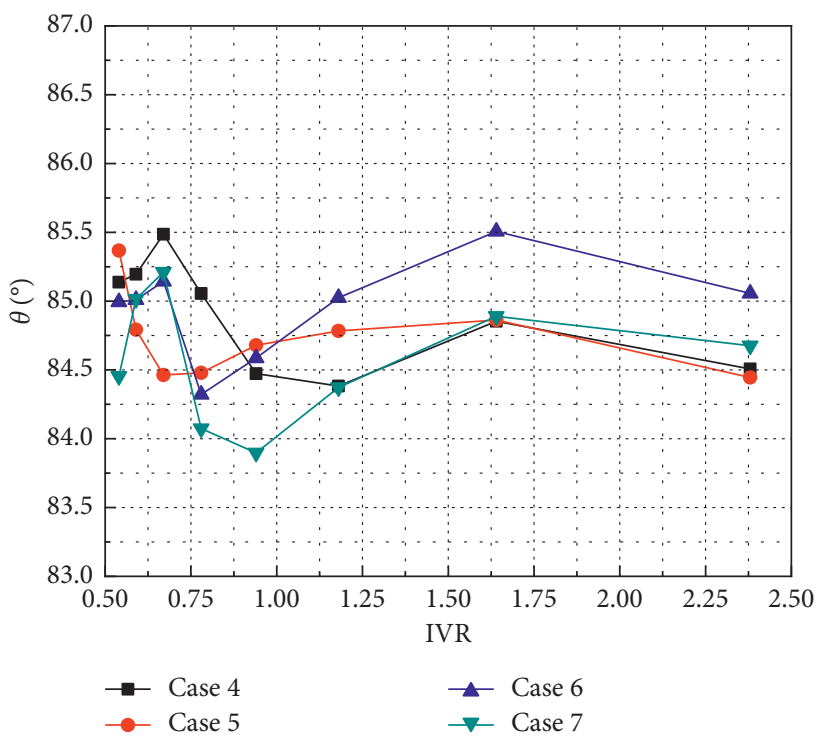

(b)

Figure 23: Hydraulic characteristics of inlet passage outlet with different dip angles. (a) Axial velocity distribution coefficient $V_{u}$. (b) Weighted-velocity average swirl angle $\theta$. 


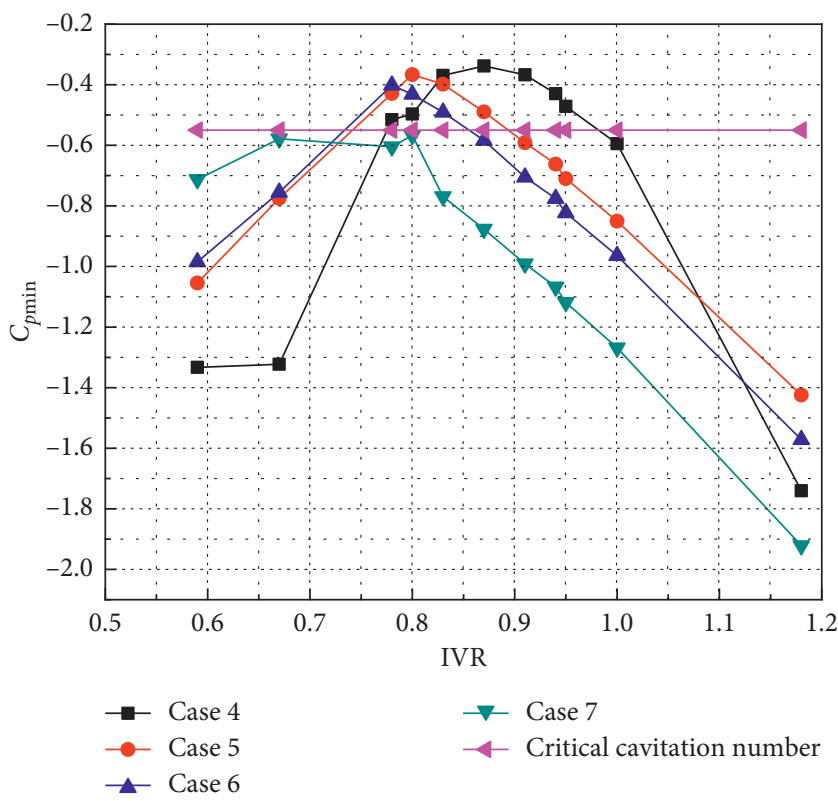

FIgUre 24: Minimum cavitation number $C_{p \min }$ with different dip angles.

TABLE 6: Evaluation index of inlet passage.

\begin{tabular}{lccr}
\hline Case & $\eta_{p}(\%)$ & $V_{u}(\%)$ & $\theta\left(^{\circ}\right)$ \\
\hline Case 4 & 95.4 & 78.1 & 84.8 \\
Case 5 & 95.7 & 77.4 & 84.6 \\
Case 6 & 95.6 & 84.1 & 84.5 \\
Case 7 & 95.2 & 85.3 & 84.0 \\
\hline
\end{tabular}

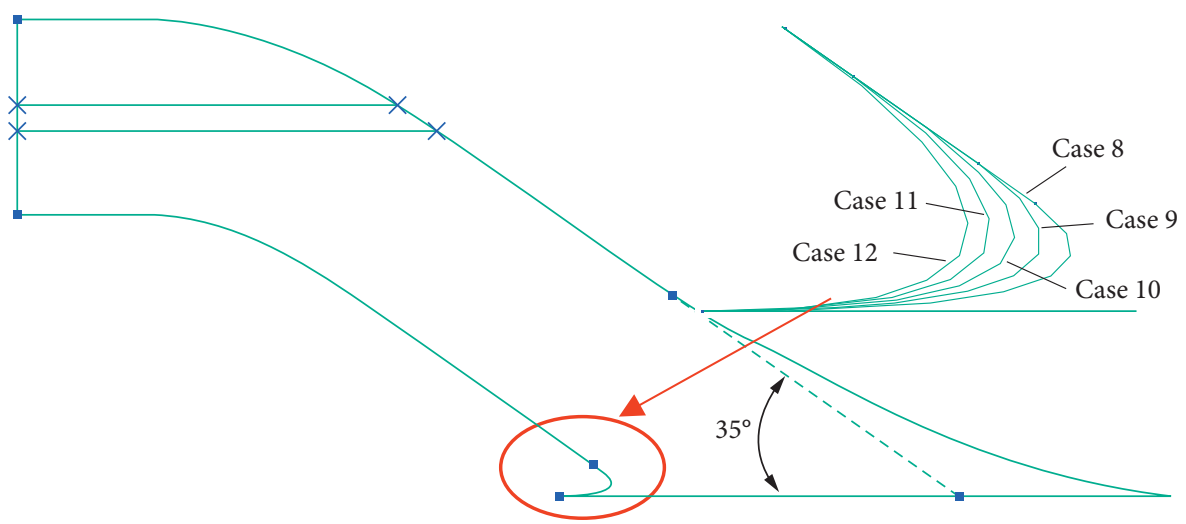

FIGURE 25: Sketch of the lip structure optimization cases.

When the dip angle of the inlet passage is in the range of $30^{\circ}-35^{\circ}$, the hydraulic performance of the inlet passage is better. If the dip angle exceeds this range, large area of wall cavitation occurs in the inlet passage and the hydraulic performance is declined. Table 6 shows the efficiency, axial velocity distribution coefficient, and weighted-velocity average swirl angle of the inlet passage for four cases at the IVR of 0.83 . The uniformity of the outlet velocity distribution of the inlet passage of Case 6 is greatly improved, and the comprehensive performance is better. Hence, on the basis of case 2, the hydraulic performance of the inlet passage is further optimized in case 6.
4.3.3. Lip Structure. The lip curvature between the smooth inlet of the horizontal inlet type inlet passage and the bottom of the ship changes most dramatically, and the change of lip curvature directly affects its hydraulic performance. The details of the lip profile still need further study. The attack angle between the flow and the lip varies greatly when the operating range of the inlet passage with fixed area is large. Figure 25 shows sketch of the lip structure optimization cases.

Figure 26 shows the variation of axial velocity distribution coefficient and weighted-velocity average swirl angle at the inlet passage outlet with IVR for different lip structure 


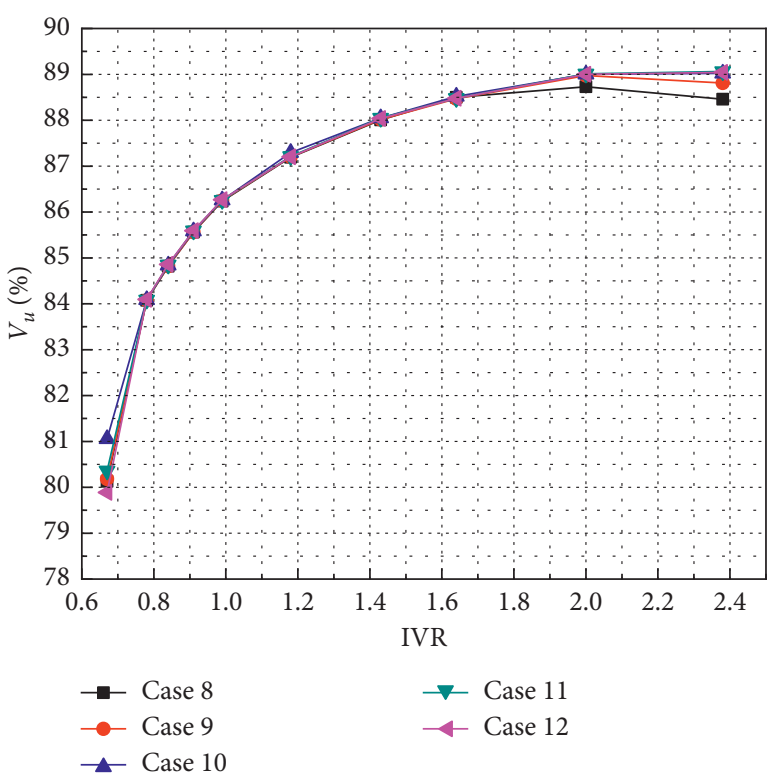

(a)

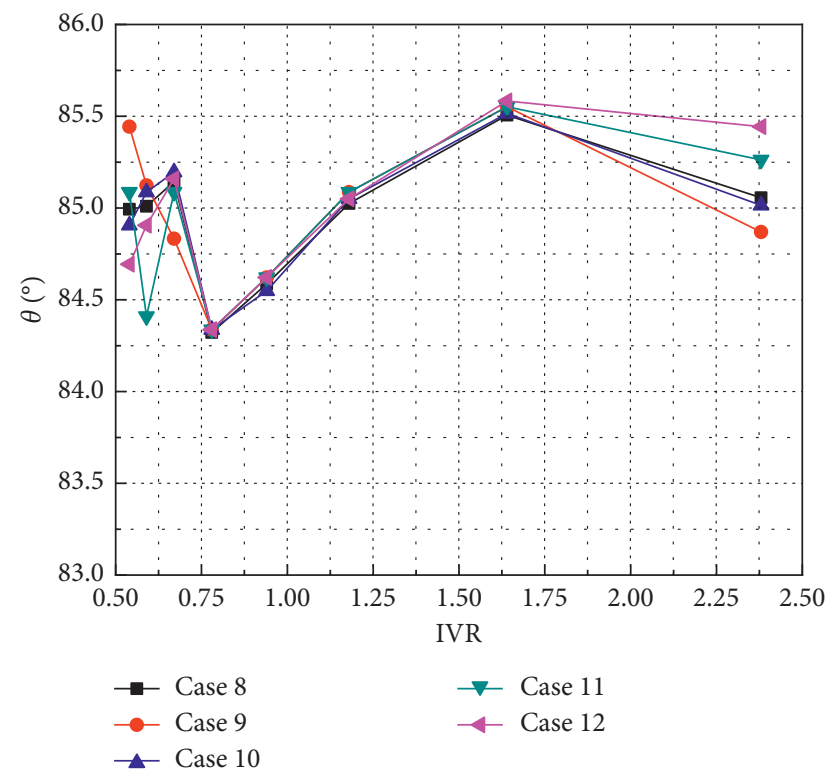

(b)

Figure 26: Hydraulic characteristics of inlet passage outlet with different lip structures. (a) Axial velocity distribution coefficient $V_{u}$. (b) Weighted-velocity average swirl angle $\theta$.

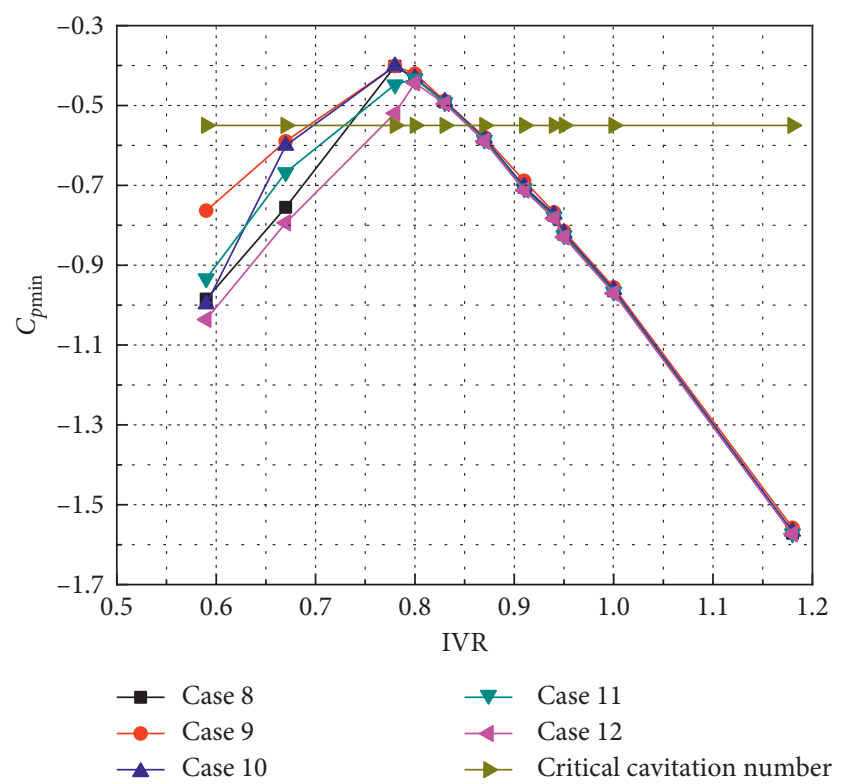

Figure 27: The minimum cavitation number $C_{p \min }$ with different lip structures.

cases. As can be seen from Figure 26(a), different lip structures have little influence on the uniformity of the inlet outlet, only slightly different under low IVR and high IVR conditions, and the overall difference is small. The outflow uniformity of the inlet passage is less affected by lip structures. Only under low IVR and high IVR conditions, the outflow uniformity of inlet passage is slightly different, and the overall difference is small. Except Case 8, the maximum axial velocity distribution coefficients of other cases are more than $88.5 \%$. Figure 26(b) shows that the difference of the weighted-velocity average swirl angle at inlet passage outlet of each case is little. The maximum difference of weighted-velocity average swirl angle at the inlet passage outlet of five cases is not more than $1.42^{\circ}$.

Figure 27 presents the variation trend of the minimum cavitation number along the wall of the inlet passage with IVR. The variation of wall pressure in different cases is basically the same between 0.8 and 2.0 of IVR. When the inlet velocity ratio is less than 0.78 , the minimum pressure on the wall of the inlet passage with different cases is quite different. Because under the low IVR condition, the sharper the lip angle, the greater the wall pressure, and the inlet passage is less likely to cavitation. The anticavitation performance of Case 9 is better. The optimal operating condition of Case 9 is the working condition of IVR 0.69-0.87.

Different lip structure cases do not change the lower edge of the lip but only the upper edge of the lip angle. From the results of the efficiency, axial velocity distribution coefficient, and weighted-velocity average swirl angle, it can be seen that the lip structure of the inlet passage affect the evaluation index slightly. Under the condition that the axial velocity distribution coefficient and weighted-velocity average swirl angle change of the different cases are small, the anticavitation performance of the inlet passage of Case 9 is better. The optimal operating conditions of Case 9 are the conditions of IVR equals $0.69-0.87$. On the basis of Case 6, the anticavitation performance of the inlet passage is further optimized in scheme 9, which widens the operating range of the inlet passage and achieves the optimization purpose. Therefore, Case 9 is the best optimization case for waterjet propulsion system without pump. The geometric parameters of Case 9 are follows: the inflow dip angle $\alpha$ is $35^{\circ}$, the length $L$ is $6.38 D_{0}$, and the upper lip angle is $4^{\circ}$. 


\section{Conclusion}

In this study, the hydraulic characteristics of the inlet passage in the waterjet propulsion system with different flow parameters and geometric parameters were studied by a combination of numerical simulation and experimental verification. In order to find the appropriate numerical settings and ensure the accuracy of the calculation, different grid numbers are used for calculation and analysis. The model test was used to verify the internal characteristics of the waterjet propulsion system, and the results show that the numerical results are in good agreement with the test results. The numerical results are reliable.

Hydraulic optimization is carried out for the prototype inlet passage from two important flow parameters, namely, inlet velocity ratio (IVR) and ship speed, and the influence of flow parameters on its hydraulic performance is analyzed. The following results are obtained by calculation and analysis:

The hydraulic performance of the inlet passage is significantly affected by the inlet velocity ratio. Under low IVR conditions, cavitation is not easy to occur in the inlet passage, but the outflow uniformity of the inlet passage is poor. Cavitation is easy to occur in the inlet passage under high IVR condition, while the outflow uniformity of the inlet passage is better. The comprehensive hydraulic performance of the inlet passage is excellent when the IVR is between 0.78 and 0.96 . The optimal operating conditions of prototype case are the conditions of IVR equals $0.78-0.96$.

There is a certain correlation between the hydraulic performance of the inlet passage and the ship speed, and the hydraulic performance of the inlet passage is limited by the ship speed. As the ship speed increases, the uniformity of the inlet passage is improved, but the cavitation range is wider. Therefore, the optimal ship speed of the prototype case is $10 \mathrm{~m} / \mathrm{s}$.

The geometry parameters of the prototype inlet passage were adjusted, and the length, dip angle, and lip structure were optimized based on CFD, and the inlet passage optimization case was obtained. Results are as follows.

The performance of the inlet passage is significantly affected by its length. The slope curvature should be considered in the design to avoid the lack of length and cause undesirable flow phenomena such as whirlpools. The hydraulic performance of the inlet passage for Case 2 is better, and the operating condition IVR is between 0.77 and 0.98 , which broadens the optimal operating conditions of the prototype case.

When the dip angle of the inlet passage is in the range of $30^{\circ}-35^{\circ}$, the hydraulic performance of the inlet passage is better. If the dip angle exceeds this range, large area of wall cavitation occurs in the inlet passage, and the hydraulic performance is declined. The uniformity of the outlet velocity distribution of the inlet passage of Case 6 is greatly improved, and the comprehensive performance is better. The optimal operating condition of Case 6 is the working condition of IVR in the range of $0.73-0.86$.

The lip structure of the inlet passage affects the evaluation index slightly. The sharper the lip angle, the better the anticavitation performance of the inlet passage. Case 9 is the best optimization case for the waterjet propulsion system without pump. The geometric parameters of Case 9 are follows: the inflow dip angle $\alpha$ is $35^{\circ}$, the length $L$ is $6.38 D_{0}$, and the upper lip angle is $4^{\circ}$. The optimal operating conditions are the conditions of IVR 0.69-0.87.

\section{Nomenclature Symbols}

\begin{tabular}{|c|c|}
\hline$u_{i}, u_{i}$ & $\begin{array}{l}\text { Velocity component of fluid in the } i \text { and } j \\
\text { directions, m/s }\end{array}$ \\
\hline$t:$ & Time, s \\
\hline$F_{i}:$ & Volume force component in the $i$ direction, $\mathrm{N}$ \\
\hline$x_{i}, x_{j}:$ & Coordinate component \\
\hline$\Delta h:$ & Hydraulic loss, m \\
\hline$g:$ & Gravitational acceleration, $\mathrm{m} / \mathrm{s}^{2}$ \\
\hline$v_{\text {passage }}:$ & $\begin{array}{l}\text { Averaged axial outflow velocity at the passage } \\
\text { outlet, } \mathrm{m} / \mathrm{s}\end{array}$ \\
\hline$V_{u}:$ & Axial velocity distribution coefficient, $\%$ \\
\hline$\overline{u_{a}}:$ & $\begin{array}{l}\text { Averaged axial velocity of the calculated section, } \\
\mathrm{m} / \mathrm{s}\end{array}$ \\
\hline$m:$ & Number of cells of the calculated section \\
\hline$P_{\text {ref }}:$ & Reference static pressure, $\mathrm{Pa}$ \\
\hline$E_{\text {inlet }}:$ & Energy at Section 3 \\
\hline$V_{1 A}:$ & Velocity of inlet capture area, $\mathrm{m} / \mathrm{s}$ \\
\hline$L:$ & Length of inlet passage, $\mathrm{m}$ \\
\hline$H:$ & Height of inlet passage, $\mathrm{m}$ \\
\hline$\rho:$ & Water density, $\mathrm{kg} / \mathrm{m}^{3}$ \\
\hline$p:$ & Pressure, $\mathrm{Pa}$ \\
\hline$\mu:$ & Dynamics viscosity coefficient \\
\hline$D_{0}:$ & Inlet diameter of the impeller, $\mathrm{mm}$ \\
\hline$\Delta P:$ & $\begin{array}{l}\text { Pressure difference between inlet and outlet of } \\
\text { inlet passage, } \mathrm{Pa}\end{array}$ \\
\hline IVR: & Inlet velocity ratio \\
\hline$v_{s}:$ & Ship speed, $\mathrm{m} / \mathrm{s}$ \\
\hline$v_{0}:$ & Design value of the fluid absolute velocity, $\mathrm{m} / \mathrm{s}$ \\
\hline$u:$ & Following velocity rating, $\mathrm{m} / \mathrm{s}$ \\
\hline$u_{a i}:$ & $\begin{array}{l}\text { Axial velocity of each element of the calculated } \\
\text { section, } \mathrm{m} / \mathrm{s}\end{array}$ \\
\hline$u_{t i}:$ & $\begin{array}{l}\text { Tangential velocity of each element of the } \\
\text { calculated section, } \mathrm{m} / \mathrm{s}\end{array}$ \\
\hline$C_{P}:$ & Pressure coefficient \\
\hline$E_{\text {Capturearea }}:$ & Energy at section $1 \mathrm{~A}$ \\
\hline$P_{\text {passage }}:$ & Pressure of inlet passage outlet, $\mathrm{Pa}$ \\
\hline$P_{1 A}:$ & Pressure of inlet capture area, $\mathrm{Pa}$ \\
\hline$\alpha:$ & Dip angle, degree \\
\hline$\eta_{\mathrm{p}}:$ & Efficiency of inlet passage, \%. \\
\hline
\end{tabular}

\section{Data Availability}

The data used to support the findings of this study are available from the corresponding author upon request.

\section{Conflicts of Interest}

The authors declare that there are no conflicts of interest regarding the publication of this paper. 


\section{Acknowledgments}

This work was supported by the National Natural Science Foundation of China (Grant no. 51779214), Peak Plan Six Talents in Jiangsu Province (Grant no. 2015-JXQC-007), Jiangsu Province 333 High Level Talents Training Project (BRA2018382), Jiangsu Planned Projects for Postdoctoral Research Funds (Grant no. 1701189B), a project funded by the Priority Academic Program Development of Jiangsu Higher Education Institutions (PAPD), Yangzhou Science and Technology Project (YZ2017289), Key Project of Water Conservancy in Jiangsu Province (Grant no. 2018042), and Yancheng New Water Source and Water Diversion Project.

\section{References}

[1] N. W. H. Bulten, Numerical Analysis of a Waterjet Propulsion System, Eindhoven University of Technology, Eindhoven, Netherlands, 2006.

[2] W.-G. Park, J. H. Jang, H. H. Chun, and M. C. Kim, "Numerical flow and performance analysis of waterjet propulsion system," Ocean Engineering, vol. 32, no. 14-15, pp. 1740-1761, 2004.

[3] T. Takai, M. Kandasamy, and F. Stern, "Verification and validation study of URANS simulations for an axial waterjet propelled large high-speed ship," Journal of Marine Science and Technology, vol. 16, no. 4, pp. 434-447, 2011.

[4] C.-J. Liu, Y.-S. Wang, Z.-H. Zhang, and J.-B. Liu, "Research on effect of different flow control volume on waterjet performance prediction," Journal of Ship Mechanics, vol. 14, no. 10, pp. 1117-1121, 2010.

[5] P. Cao, Y. Wang, C. Kang, G. Li, and X. Zhang, "Investigation of the role of non-uniform suction flow in the performance of water-jet pump," Ocean Engineering, vol. 140, pp. 258-269, 2017.

[6] M.-C. Kim, W.-G. Park, H.-H. Chun, and U.-H. Jung, "Comparative study on the performance of Pod type waterjet by experiment and computation," International Journal of Naval Architecture and Ocean Engineering, vol. 2, no. 1, pp. 1-13, 2010.

[7] D. R. Lavis, B. G. Forstell, and J. G. Purnell, "Compact waterjets for high-speed ships," Ships and Offshore Structures, vol. 2, no. 2, pp. 115-125, 2007.

[8] A. Eslamdoost, L. Larsson, and R. Bensow, "A pressure jump method for modeling waterjet/hull interaction," Ocean Engineering, vol. 88, pp. 120-130, 2014.

[9] C. J. Liu, "Influence of hull waterjet interaction on propulsion performances of waterjet," Journal of Shanghai Jiaotong University, vol. 50, no. 1, pp. 91-97, 2016.

[10] R. Verbeek and N. Bulten, "Recent development in waterjet design," in Proceedings of the International Conference on Waterjet Propulsion II, RINA., Amsterdam, Netherlands, 1998.

[11] C. Wang, X. He, W. Shi, X. Wang, X. Wang, and N. Qiu, "Numerical study on pressure fluctuation of a multistage centrifugal pump based on whole flow field," AIP Advances, vol. 9, no. 3, Article ID 035118, 2019.

[12] Z. Luo, Z.-J. Chen, C.-S. Sun, and H.-F. Ai, "Vortex-induced effect and structural strength of the channel-grating in waterjet propulsion," Journal of Mechanical Engineering, vol. 29, no. 2, pp. 19-22, 2007.

[13] S. Wang, Y. Wang, S. Jin, and J. Ding, "Effect of inlet grid on duct flow performance and waterjet propulsion performance," Journal of Mechanical Engineering, vol. 49, no. 14, pp. 164-169, 2013.

[14] S. P. Chang, Y. S. Wang, Z. Y. Pang et al., "The hydrodynamic force analysis and strength calculation of waterjet intake grid," Ship Science and Technology, vol. 31, no. 1, pp. 110-114, 2010.

[15] A. Papanikolaou, "Machinery installation, propulsion and steering devices," in Ship Design, Springer, Berlin, Germany, 2014.

[16] M.-C. Fang, J.-H. Luo, and M.-L. Lee, "A nonlinear mathematical model for ship turning circle simulation in waves," Journal of Ship Research, vol. 49, no. 2, pp. 69-79, 2005.

[17] J. Pandey and K. Hasegawa, "Study on turning manoeuvre of catamaran surface vessel with a combined experimental and simulation method," IFAC-PapersOnLine, vol. 49, no. 23, pp. 446-451, 2016.

[18] S. Gao, Q. D. Zhu, and L. Li, "Study on the motion control technology based on double water jet propulsion ships," Ship Engineering, vol. 30, no. 3, pp. 60-63, 2008.

[19] R. Deng, F. Kong, and H. D. Kim, "Numerical simulation of fluidic thrust vectoring in an axisymmetric supersonic nozzle," Journal of Mechanical Science and Technology, vol. 28, no. 12, pp. 4979-4987, 2014.

[20] A. Banazadeh, F. Saghafi, M. Ghoreyshi, and P. Pilidis, "Experimental and computational investigation into the use of co-flow fluidic thrust vectoring on a small gas turbine," The Aeronautical Journal, vol. 112, no. 1127, pp. 17-25, 2008.

[21] H. Fangfang, W. Peng, W. Dazhuan, and W. LeQin, "Numerical study on the stall behavior of a water jet mixed-flow pump," Journal of Marine Science and Technology, vol. 19, no. 4, pp. 438-449, 2014.

[22] F. Liu, "Study on the ship's loss-speed in wind and waves," Journal of Dalian Marine College, vol. 4, no. 18, pp. 347-351, 1992.

[23] L. Cheng and W. Qi, "Rotating stall region of water-jet pump," Transactions of Famena, vol. 38, no. 2, pp. 31-40, 2014.

[24] C. Xia, L. Cheng, C. Luo, W. Jiao, and D. Zhang, "Hydraulic characteristics and measurement of rotating stall suppression in a waterjet propulsion system," Transactions of Famena, vol. 4, no. 42, pp. 85-100, 2018.

[25] L. Tan, Z. Yu, Y. Xu, L. Yabin, and C. Shuliang, "Role of blade rotational angle on energy performance and pressure fluctuation of a mixed-flow pump," Proceedings of the Institution of Mechanical Engineers, Part A: Journal of Power and Energy, vol. 231, no. 3, pp. 227-238, 2017.

[26] L. Tan, Z. F. Xie, Y. B. Liu, and H. Yue, "Influence of T-shape tip clearance on performance of a mixed-flow pump," Proceedings of the Institution of Mechanical Engineers, Part A: Journal of Power and Energy, vol. 232, no. 4, pp. 386-396, 2018.

[27] X. He, W. Jiao, C. Wang, and W. Cao, "Influence of surface roughness on the pump performance based on computational fluid dynamics," IEEE Access, vol. 7, no. 1, pp. 105331-105341, 2019.

[28] C. Wang, B. Hu, Y. Zhu, X. Wang, C. Luo, and L. Cheng, "Numerical study on the gas-water two-phase flow in the selfpriming process of self-priming centrifugal pump," Processes, vol. 7, no. 6, p. 330, 2019.

[29] Y. Liu and L. Tan, "Spatial-temporal evolution of tip leakage vortex in a mixed flow pump with tip clearance," Journal of Fluids Engineering, vol. 141, no. 8, Article ID 081302, 2019.

[30] Y. Liu and L. Tan, "Tip clearance on pressure fluctuation intensity and vortex characteristic of a mixed flow pump as turbine at pump mode," Renewable Energy, vol. 129, pp. 606-615, 2018. 
[31] Y. Hao and L. Tan, "Symmetrical and unsymmetrical tip clearances on cavitation performance and radial force of a mixed flow pump as turbine at pump mode," Renewable Energy, vol. 127, pp. 368-376, 2018.

[32] P. C. Chin, Water Jet Propulsion of the Vessel, National Defence Industrial Press, Beijing, China, 1986.

[33] W.-G. Park, H. S. Yun, H. H. Chun, and M. C. Kim, "Numerical flow simulation of flush type intake duct of waterjet," Ocean Engineering, vol. 32, no. 17-18, pp. 2107-2120, 2005.

[34] J.-M. Ding and Y.-S. Wang, "Research on flow loss of inlet duct of marine waterjets," Journal of Shanghai Jiaotong University (Science), vol. 15, no. 2, pp. 158-162, 2010.

[35] C. Wang, W. Shi, X. Wang et al., "Optimal design of multistage centrifugal pump based on the combined energy loss model and computational fluid dynamics," Applied Energy, vol. 187, pp. 10-26, 2017.

[36] K. H. Jung, K. C. Kim, S. Y. Yoon, S. H. Kwon, H. H. Chun, and M. C. Kim, "Investigation of turbulent flows in a waterjet intake duct using stereoscopic PIV measurements," Journal of Marine Science and Technology, vol. 11, no. 4, pp. 270-278, 2006.

[37] J. Gong, C.-Y. Guo, T.-C. Wu, and D.-G. Zhao, "Particle image velocimetry measurement of velocity distribution at inlet duct of waterjet self-propelled ship model," Journal of Hydrodynamics, vol. 29, no. 5, pp. 879-893, 2017.

[38] D. Bonaiuti, M. Zangeneh, R. Aartojarvi, and J. Eriksson, "Parametric design of a waterjet pump by means of inverse design, CFD calculations and experimental analyses," Journal of Fluids Engineering, vol. 132, no. 3, Article ID 031104, 2010.

[39] C. Wang, X. He, D. Zhang, B. Hu, and W. Shi, "Numerical and experimental study of the self-priming process of a multistage self-priming centrifugal pump," International Journal of Energy Research, vol. 43, no. 9, pp. 4074-4092, 2019.

[40] W. X. Jiao, L. Cheng, J. Xu, and C. Wang, "Numerical analysis of two-phase flow in the cavitation process of a waterjet propulsion pump system," Processes, vol. 7, no. 10, p. 690, 2019.

[41] Waterjet propulsive performance prediction-waterjet inlet passage, pump loop and waterjet system tests and extrapolation," in Proceedings of the Final Recommendations of the Specialist Committee on Validation of Waterjet Test Procedures to the 24th ITTC, ITTC, Edinburgh, UK, 2005.

[42] D. Keegan and D. Martin, "Use of RANS for waterjet analysis of a high-speed sealift concept vessel," in Proceedings of the First International Symposium on Marine Propulsors, Trondheim, Norway, 2009.

[43] F. Q. Yu, J. M. Ding, W. G. Yang et al., "Research on stream tube and capture area of waterjet propulsion system," Ship Science and Technology, vol. 31, no. 4, pp. 54-57, 2009. 


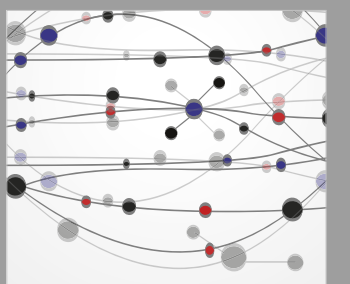

The Scientific World Journal
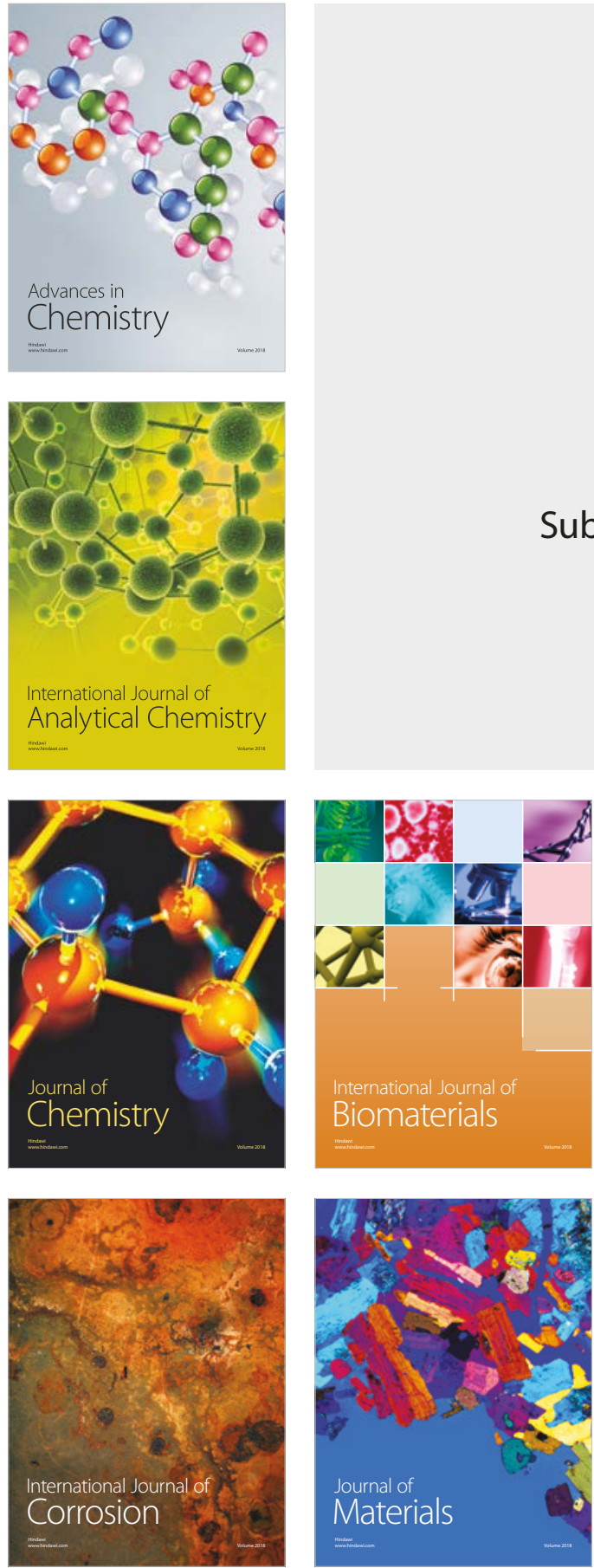

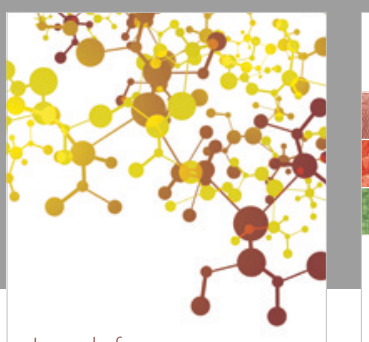

Journal of

Applied Chemistry
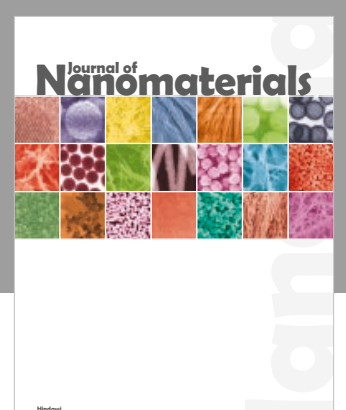

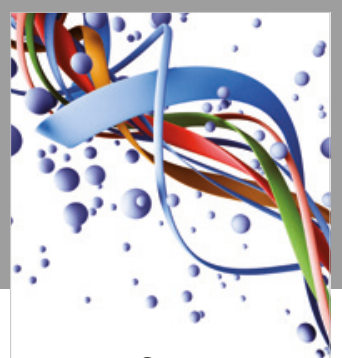

Scientifica

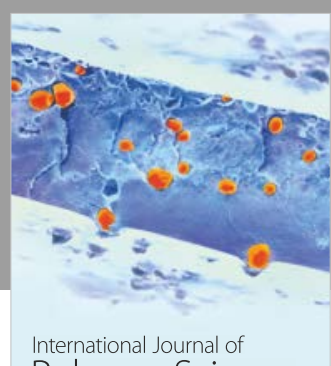

Polymer Science

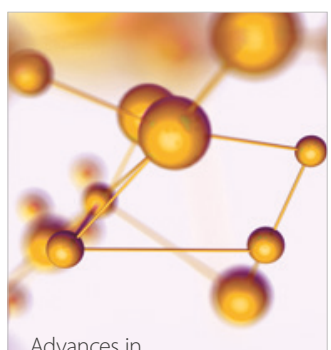

Physical Chemistry
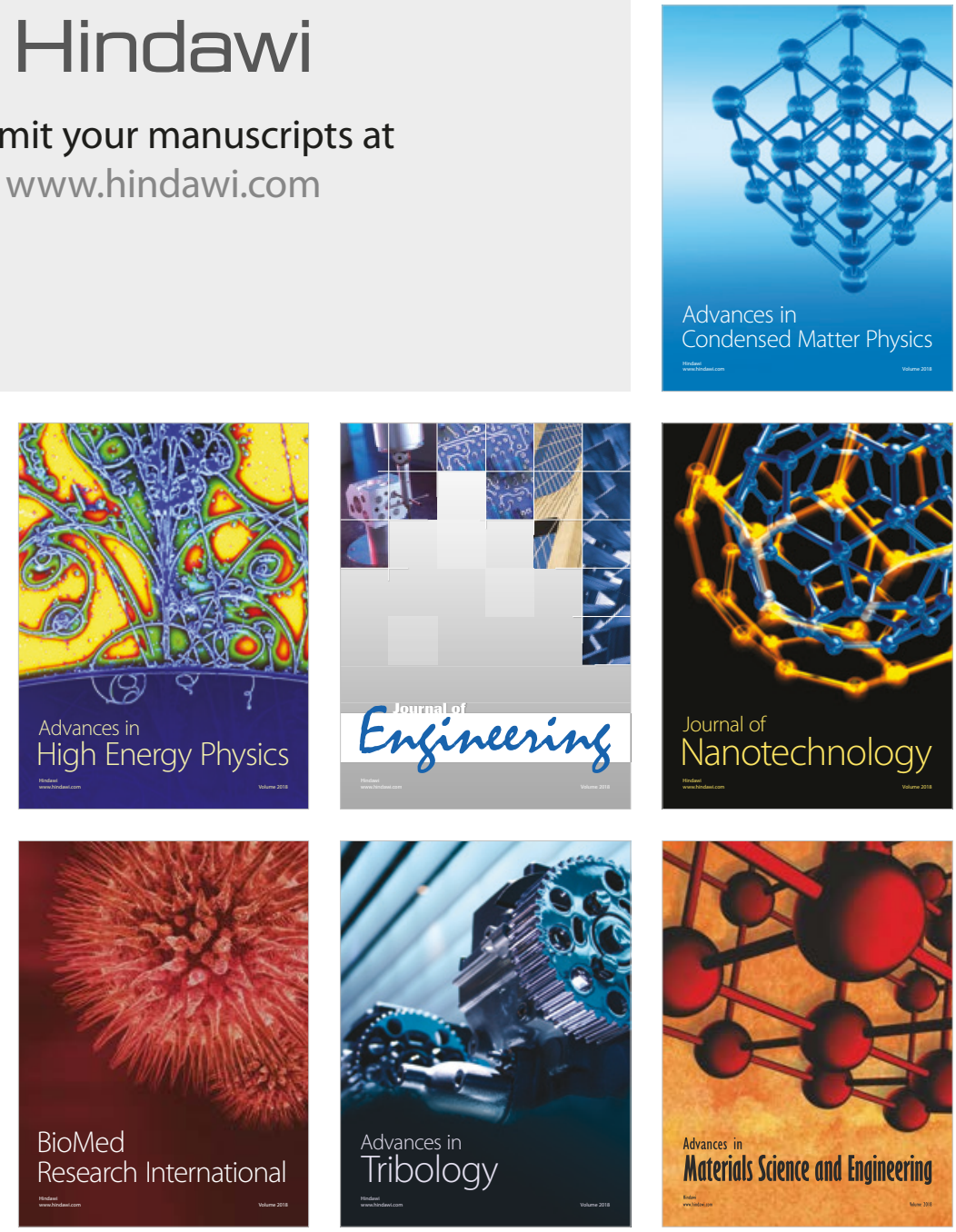